\title{
Rapid Imaging: Recent Advances in Abdominal MRI for Reducing Acquisition Time and Its Clinical Applications
}

\author{
Jeong Hee Yoon, $\mathrm{MD}^{1}$, Marcel Dominik Nickel, $\mathrm{PhD}^{2}$, Johannes M. Peeters, $\mathrm{PhD}^{3}$, Jeong Min Lee, $\mathrm{MD}^{1,4}$ \\ ${ }^{1}$ Department of Radiology, Seoul National University Hospital, Seoul National University College of Medicine, Seoul, Korea; ${ }^{2}$ MR Application \\ Predevelopment, Siemens Healthcare GmbH, Erlangen, Germany; ${ }^{3}$ MR Clinical Science, Philips Healthcare, Best, The Netherlands; ${ }^{4}$ Institute of \\ Radiation Medicine, Seoul National University Medical Research Center, Seoul, Korea
}

Magnetic resonance imaging (MRI) plays an important role in abdominal imaging. The high contrast resolution offered by MRI provides better lesion detection and its capacity to provide multiparametric images facilitates lesion characterization more effectively than computed tomography. However, the relatively long acquisition time of MRI often detrimentally affects the image quality and limits its accessibility. Recent developments have addressed these drawbacks. Specifically, multiphasic acquisition of contrast-enhanced MRI, free-breathing dynamic MRI using compressed sensing technique, simultaneous multi-slice acquisition for diffusion-weighted imaging, and breath-hold three-dimensional magnetic resonance cholangiopancreatography are recent notable advances in this field. This review explores the aforementioned state-of-theart techniques by focusing on their clinical applications and potential benefits, as well as their likely future direction. Keywords: MRI; Compressed sensing; Sparse; Simultaneous multi-slice; GRASP; Free breathing; MRCP; Diffusion weighted imaging; GRASE

\section{INTRODUCTION}

In recent years, magnetic resonance imaging (MRI) has been widely used for abdominal imaging. The enhanced soft tissue contrast of MRI has improved lesion detection in abdominal organs. In addition, its capability of providing multiparametric images has greatly assisted in the characterization of lesions and monitoring of treatment response. The performance of MRI has led to it being perceived as a problem-solving imaging modality.

Received December 31, 2018; accepted after revision July 22, 2019.

This study was supported by grant 04-2017-0800 from the Seoul National University Hospital Research Fund.

Corresponding author: Jeong Hee Yoon, MD, Department of Radiology, Seoul National University Hospital, Seoul National University College of Medicine, 101 Daehak-ro, Jongno-gu, Seoul 03080, Korea.

- Tel: (822) 2072-2293 - Fax: (822) 743-6385

- E-mail:jhjhry@gmail.com

This is an Open Access article distributed under the terms of the Creative Commons Attribution Non-Commercial License (https://creativecommons.org/licenses/by-nc/4.0) which permits unrestricted non-commercial use, distribution, and reproduction in any medium, provided the original work is properly cited.
However, compared with computed tomography (CT) or ultrasonography, MRI requires a long scan time, which often limits its clinical applicability. For abdominal MRI, this lengthy acquisition time often induces motion artifacts, which can significantly hinder image quality. Respiratorytriggering or gating have been used for some sequences, but these techniques do not completely eliminate motion artifacts. Furthermore, they are not feasible to use in dynamic contrast-enhanced MRI, due to scan inefficiency. In addition, the longer acquisition time, often causes MRI to fail in capturing the optimal phase during dynamic phase acquisition. In particular, for liver MRI scans using gadoxetic acid, because the arterial window is relatively short, effective capturing of the arterial phase has been a troublesome issue for radiologists $(1,2)$. Efforts have been made to accelerate the scanning speed of MRI, including by making improvements to the hardware related to aspects such as gradient slew rate and amplitude; and also to expand the application of various types of parallel imaging $(3,4)$ and the adoption of other techniques, such as view-sharing down to keyhole imaging (5-11). Recently, compressed sensing has been introduced in abdominal MRI to improve scan speed, in combination with 
parallel imaging (12-14). In this review, we present a brief overview of compressed sensing, focusing on its clinical application in abdominal MRI, in addition to other recently implemented strategies aimed at reducing scan time for diffusion-weighted imaging (DWI) and magnetic resonance cholangiopancreatography (MRCP).

\section{Parallel Imaging and Compressed Sensing to Accelerate Scan Speed in Abdominal MRI}

\section{Parallel Imaging}

The long acquisition time of MRI is closely related with $\mathrm{k}$-space sampling. If it were possible to reconstruct images with partially sampled k-space data, it would improve the temporal resolution of MRI. To reduce the scan time, parallel imaging is often applied. Parallel imaging involves simultaneous data acquisition via the multiple receiver coil elements of phase array coils, a feature available on most clinical scanners; this results in data redundancy. Phased array coils acquire data from multiple elements simultaneously, and variation in sensitivity profiles among those elements can then be exploited in image reconstruction.

For conventional parallel imaging, the required phaseencoding steps are undersampled on a regular sublattice. In other words, k-space is undersampled by skipping phaseencoding lines in equidistant steps (Fig. 1). This violates the Nyquist criterion and results in aliasing artifacts in a naïve zero-padded reconstruction, and affects image quality and diagnostic performance. Aliasing artifacts are mitigated by parallel imaging reconstruction, which can be done in image $(x, y)$, k-space $(k x, k y)$, or in hybrid $(x$, ky) domains (Fig. 2A). The methods involved are classified into two categories, depending on where the images are reconstructed and artifacts are corrected: image domain or k-space domain. The most commonly used techniques in the clinical field are sensitivity encoding (SENSE), which performs post-Fourier transformation in the image domain (4) and generalized autocalibrating partially parallel acquisition (GRAPPA), which works in the k-space domain (3). The former uses coil sensitivity maps to disentangle aliased data, while the latter exploits correlations among neighboring $\mathrm{k}$-space lines and coil elements to calculate weights by which non-acquired k-space data are reconstructed. SENSE has the advantage of numerical efficiency, but is prone to a mismatch of reference data and scan data. Thus, SENSE requires compensatory strategies to overcome the possible mismatch (15). Conversely, GRAPPA usually uses auto-calibration and deals with the mismatch between measured and reference data, but it has a lower numerical efficiency than SENSE. Parallel imaging is wellestablished for both two-dimensional (2D) and threedimensional (3D) Cartesian data acquisitions. It can also be extended to non-Cartesian samplings with the underlying concept being to utilize coil sensitivity data or correlations between different data from different coil elements. Indeed, parallel imaging has been shown to improve abdominal MRI through efficient $k$-space sampling, thereby improving spatial resolution, and reducing acquisition time and sensitivity to motion $(3,4,16,17)$.

However, the acceleration factor, specified by the ratio of fully sampled k-space data to undersampled k-space data, is limited by several factors. A high acceleration factor may cause a decreased signal-to-noise ratio, additional noise amplification quantified by the geometry factor due to the

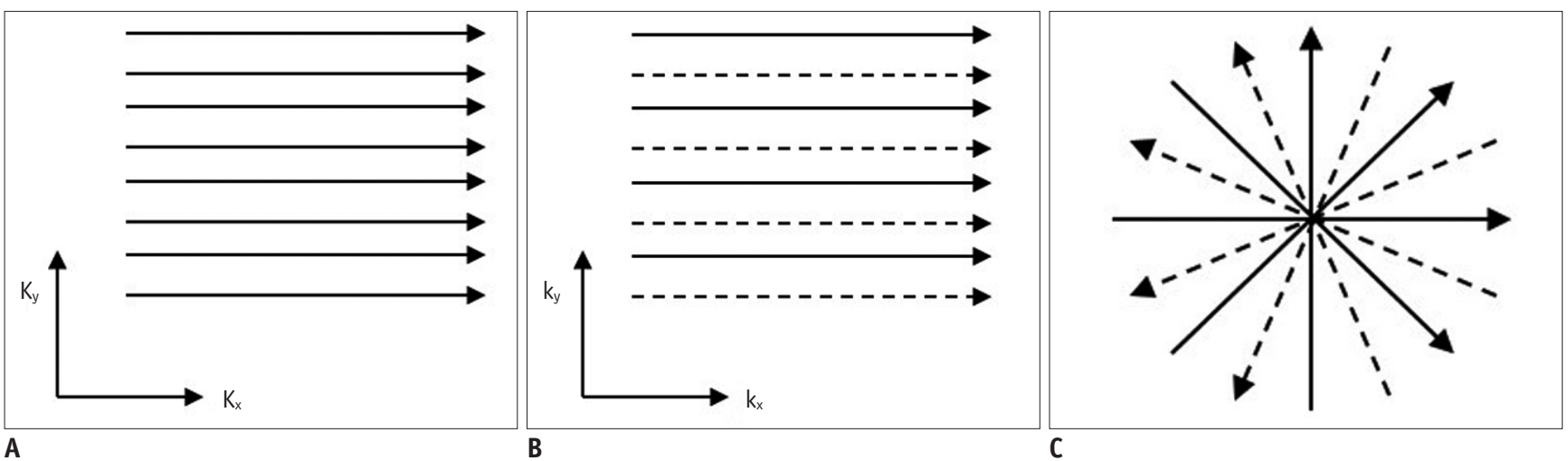

Fig. 1. Different k-space sampling schemes.

Cartesian acquisition with fully sampled k-space (A), uniformly undersampled with acceleration factor of 2 (B), or non-Cartesian acquisition with skipping spokes in radial acquisition (C). Solid and dashed lines refer to acquired and skipped $k$-space data, respectively. $\mathrm{K}_{\mathrm{x}}=$ frequency encoding direction, $\mathrm{K}_{\mathrm{y}}=$ phase encoding direction 
coil setup, and remaining aliasing artifacts. Consequently, it is often challenging to achieve stable and acceptable image quality for acceleration factors larger than 4 in clinical practice.

\section{Compressed Sensing}

For further acceleration of MR acquisition speed, the concept of compressed sensing has been investigated (12); this relies on the premise that a natural image is compressible. Based on our long-standing experience with using picture archiving and communication systems, we can safely assume that medical images are also compressible, without loss of essential information or degradation of image quality. This also implies redundancy in MRI scans. If most acquired data can be discarded without perceptual loss, there is no need to acquire unnecessary data in the first place, and exploiting the redundancy of MRI data would mean that fewer samples should be sufficient for

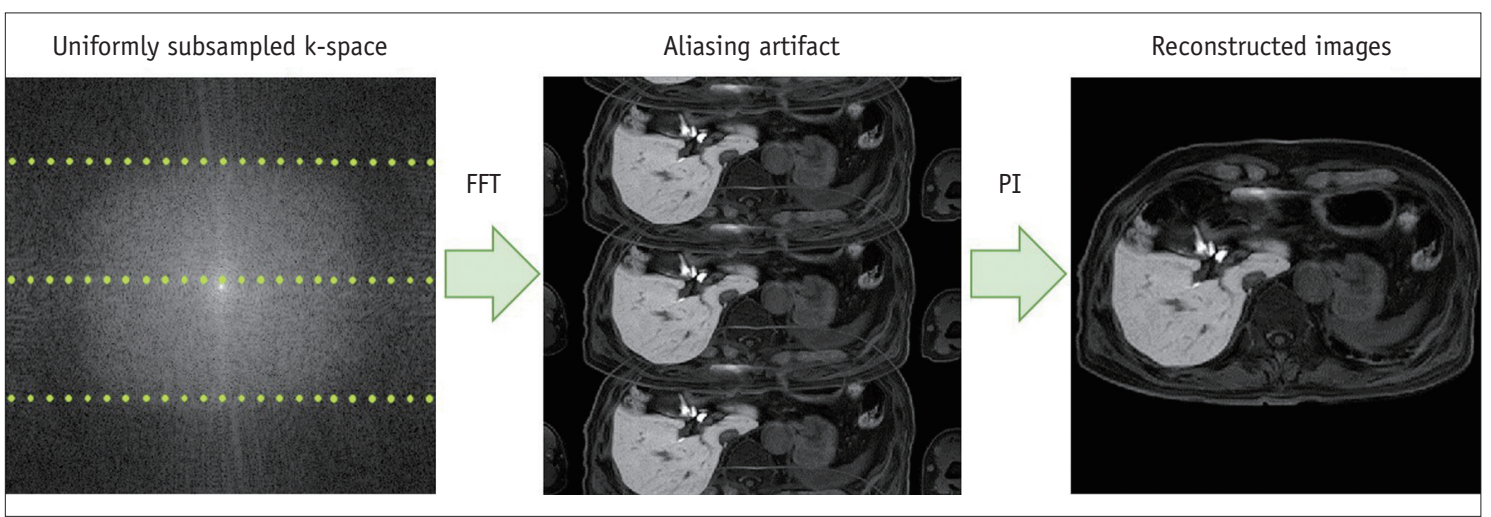

A

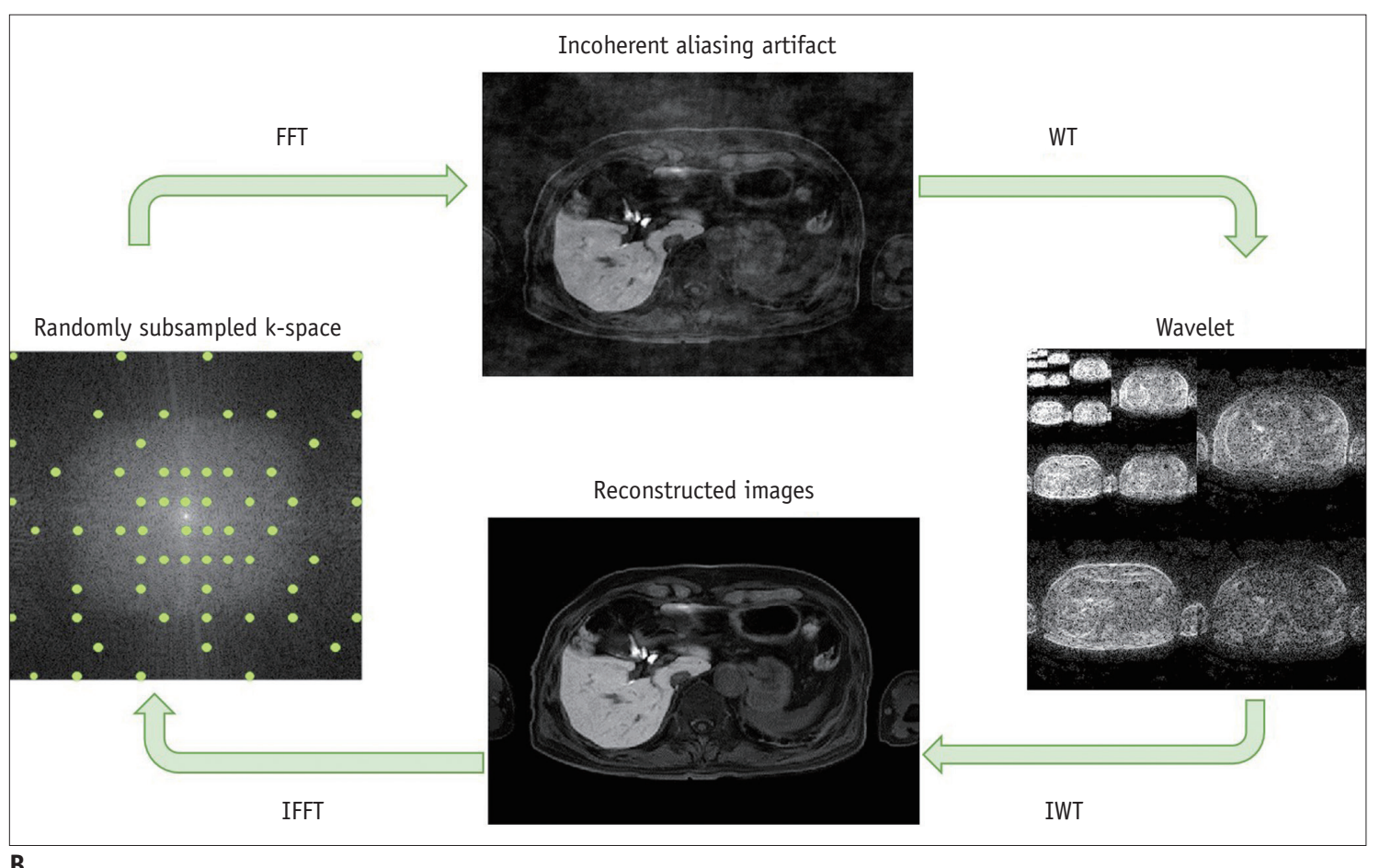

Fig. 2. Graphical representation of principles of parallel imaging and compressed sensing.

In parallel imaging (A), uniform subsampling gives typical aliasing artifacts. Parallel imaging reconstruction makes it possible to achieve aliasfree image. For SENSE, parallel imaging reconstruction is done in image space, whereas de-aliasing is already done before FFT in generalized autocalibrating partially parallel acquisitions (GRAPPA). In compressed sensing (B), variable-density, pseudo-random subsampling produces incoherent noise-like aliasing artifact after FFT. Sparsity (in this case, wavelet) transform allows setting of sparsity constraints. Image is obtained after IWT into image domain. IFFT back to k-space allows data consistency checking. After several iterations, final image is delivered with optimal balance between data consistency and sparsity constraints. FFT = Fourier transform, IFFT = inverse Fourier transform, IWT $=$ inverse transform, PI = parallel imaging, SENSE = sensitivity encoding, WT = wavelet transform 
reconstructing images with relevant information, thereby reducing the scan time. In contrast, compression of medical images after acquisition does not change the acquisition time. However, the application of compressed sensing for MRI acquisition has been slow, because it is not clear which parts of the signals contain essential information and which parts are redundant.

Compressed sensing requires three conditions: sparsity, incoherence, and non-linear reconstruction $(12,14,18)$. Sparsity refers to a condition in which only a small number of coefficients carries the relevant information in images in a suitable transform domain. Strong sparsity, i.e., involving only a few non-vanishing coefficients, is desired to achieve higher compression. As described earlier, MRI is inherently sparse in its transform domain. Even though an image may be sparse, the challenge remains to find the non-vanishing components from any image with a fixed acquisition scheme. The basis of compressed sensing is choosing samplings that have sufficient overlap with any sparse representation in the transform domain. An incoherent and random sampling scheme achieves this requirement, because the associated aliasing artifacts are noise-like, and thus thresholding in the transform domain allows identification of relevant coefficients $(12,14,18)$. In practice, however, pure random sampling is not likely to be feasible, because of hardware or physiological constraints, such as slew rate, eddy currents, and nerve stimulation (12, 14). Consequently, pseudo-random sampling patterns are often used $(14,19)$. Figure $2 B$ illustrates the process of image acquisition using compressed sensing.

As with parallel imaging, compressed sensing can be combined with various sequences, including Cartesian and non-Cartesian acquisition schemes (20-24). In particular, non-Cartesian sampling schemes have several advantages for compressed sensing. The typical aliasing artifacts from radial or spiral sampling schemes in non-Cartesian sequences are less coherent than those of the regular undersampling used for Cartesian parallel imaging, and therefore comply more naturally with the prerequisites $(14,18)$. In addition, central k-space is inherently densely sampled and it contributes to a better signal-to-noise ratio, even when acquisition time is reduced.

The final prerequisite for compressed sensing is non-linear reconstruction, which is necessary to determine the sparse representation discussed above $(14,18)$. The strategy of image reconstruction provides a balance between data consistency and sparsity, typically through optimizing the following cost function $(14,18)$ :

$$
\hat{\chi}=\operatorname{argmin}_{\chi}\|y-A \chi\|_{2}^{2}+\lambda\|\Psi \chi\|_{1}
$$

Here, $\hat{\chi}$ is the reconstructed image, $y$ represents the acquired $\mathrm{k}$-space data, and $\mathrm{A}$ is the system operator that maps the image $\chi$ to the $k$-space data. The system operator A includes information about the k-space trajectory, the coil sensitivities, and Fourier transformation. Furthermore, $\Psi$ is a sparsifying transformation applied to the image and $\lambda$ is the regularization factor. The first term ensures data consistency and is identical to the term in a non-regularized SENSE reconstruction. The second term enforces sparsity in the transform domain though the chosen 11 norm. The regularization factor $(\lambda)$ balances the data consistency and data sparsity (Fig. 3 ).

Unlike parallel imaging, compressed sensing uses sparsity to reduce the number of required phase-encoding steps, which are independent of the coil setup. However, as shown by the equation above, it can be naturally combined with parallel imaging. Thus, a combination of compressed
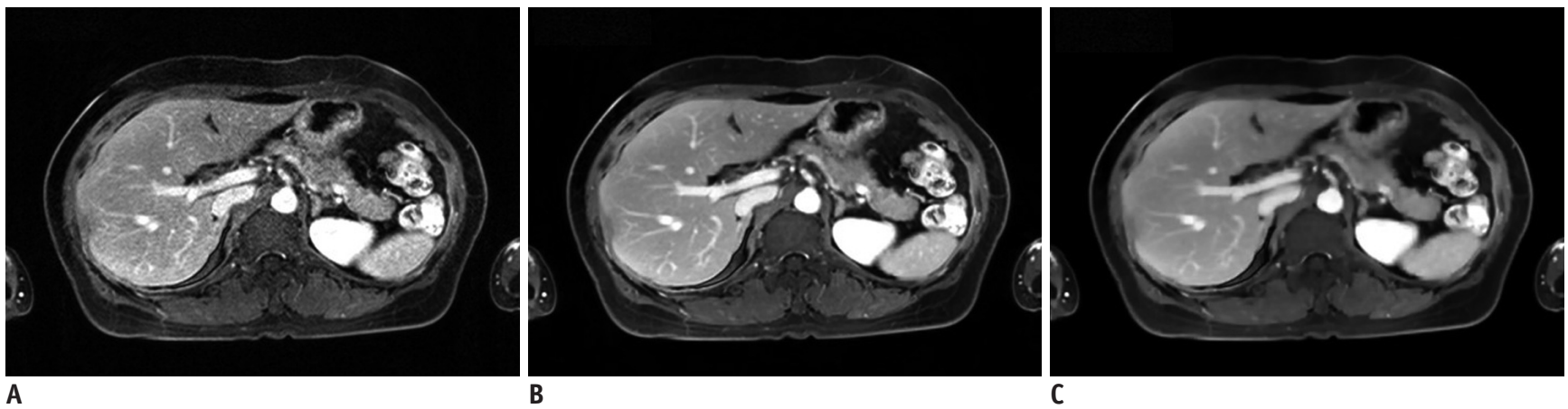

Fig. 3. Effect of regularization parameters.

Same dataset was reconstructed using no regularization parameter (A), suggested regularization parameter (B), and 10-fold higher regularization parameter than that suggested (C). Images show different imaging textures and signal-to-noise ratios, according to regularization parameters. 
sensing and parallel imaging tends to accelerate the MRI speed more than does parallel imaging alone (25). In addition, this combination reduces the risk of losing small coefficients (and in turn low-contrast objects) and of temporal or spatial blurring.

\section{Clinical Applications}

\section{Current Issues Related to Obtaining Dynamic 3D T1-Weighted Imaging}

As mentioned above, the extended acquisition time is a limiting factor for abdominal imaging, and this often causes motion artifacts in breath-hold examinations. It is particularly troublesome for dynamic T1-weighted imaging, which is the most important sequence for lesion detection and characterization. For liver MRI using a hepatocytespecific contrast agent, in particular, transient motion often occurs and results in motion artifacts at critical time points $(26,27)$. Furthermore, compared with extracellular contrast media, a shorter arterial time window challenges the acquisition of optimal arterial phase imaging. The incidence of motion artifacts due to limited breath-hold capacity or transient motion is known to be reduced by shortening the acquisition time (28). For this purpose, multiple arterial phase images are widely performed for liver MRI $(2,9)$. Although parallel imaging has improved the temporal resolution, multi-arterial phase often achieves a high temporal resolution at the expense of spatial resolution; and this may negatively affect image quality, and potentially diagnostic performance. For further acceleration of MRI while balancing both types of resolution, other strategies have been suggested including view-sharing down to keyhole techniques $(9,10)$. Such strategies have been applied to dynamic contrast-enhanced sequences to acquire an optimal arterial phase for liver MRI $(9,10)$. However, it is possible that all phases may be degraded due to motion and that temporal blurring may occur because sampled data are shared throughout the phases. Hence, there has been an ongoing attempt to achieve optimal arterial phase timing without significant motion artifacts.

\section{D T1-Weighted Images with High Temporal Resolution}

\section{Static Imaging}

Compressed sensing with parallel imaging acquisition can achieve higher acceleration for static imaging by using sparsity in the spatial domain. Using a combination of compressed sensing and parallel imaging allows acceleration by a factor greater than four (29), and the acquisition time can be reduced to less than 10 seconds, without significant compromise of image quality (Fig. 4). High temporal resolution of breath-hold sequences can be helpful for patients with limited breath-holding capacity.

\section{Dynamic Imaging}

Contrast-enhanced dynamic sequence is a good application of compressed sensing, because sparsity in the temporal domain can additionally be exploited. Achieving T1-weighted dynamic images with high temporal resolution has been attempted using compressed sensing and either
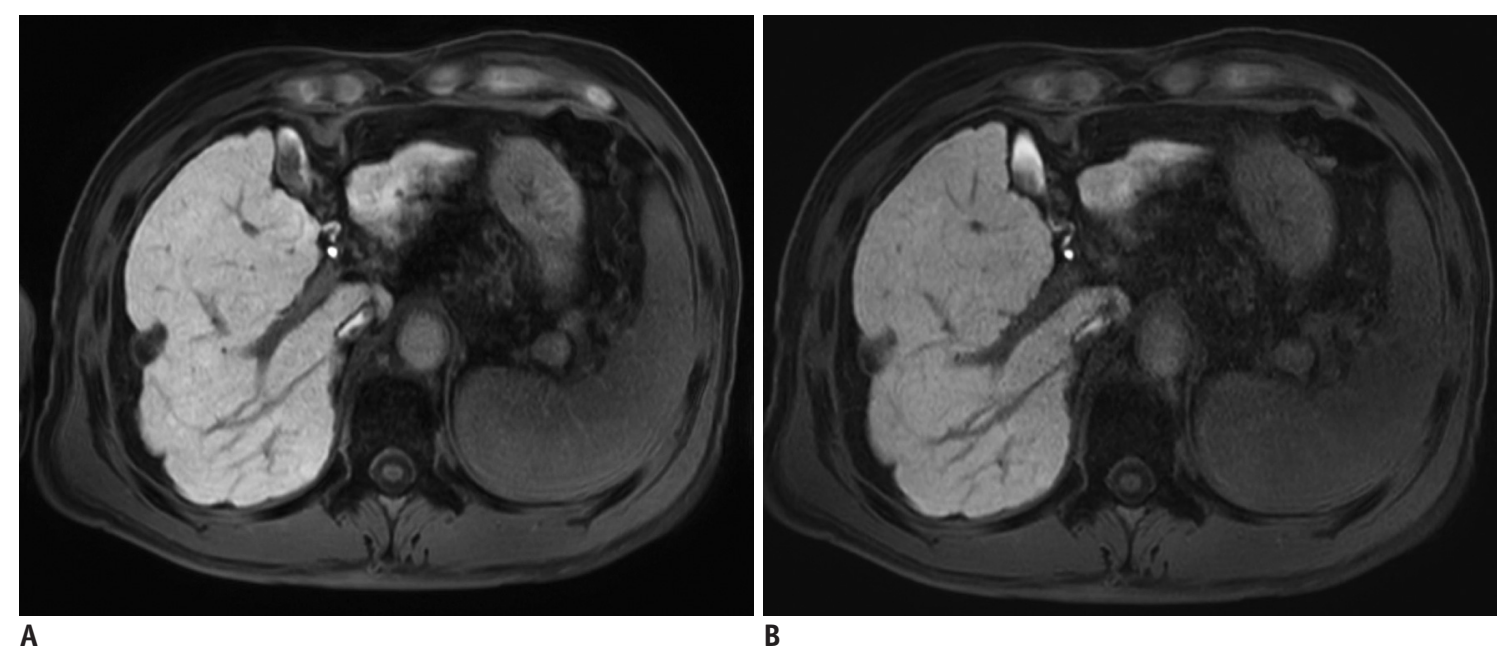

Fig. 4. Hepatobiliary phase of gadoxetic acid-enhanced MRI in 69-year-old man.

A. First image was obtained with parallel imaging alone (SENSE) with acceleration factor of 2.8. B. Next image was acquired using compressed sensing and SENSE with acceleration factor of 7.17. Although both images show comparable image quality and spatial resolution (reconstruction voxel size $0.99 \times 0.99 \times 3 \mathrm{~mm}$ ), image acquisition time was 15 seconds in (A) and 6 seconds in (B). 
Cartesian or non-Cartesian acquisition.

Cartesian sampling schemes should follow a pseudorandom, underdamping pattern to utilize compressed sensing (18, 30-32). Multiple arterial phase acquisitions can be obtained in a single breath-hold. This is similar to previous studies in which dual or triple arterial phase acquisitions were obtained in a single breath-hold $(2,9,10$, 28). However, this combination of compressed sensing and parallel imaging allows both higher temporal and spatial resolution than does multi-arterial phase acquisition using parallel imaging only (Fig. 5) (2). In addition, it minimizes the concerns about temporal blurring with appropriate reconstruction algorithms and the contamination by motion artifacts as compared to view-sharing techniques (Figs. $6,7)(9,10,28)$. If high temporal resolution is critical, view-sharing techniques can be combined with compressed sensing and parallel imaging for further acceleration of scan speed (33). As parallel imaging, a combination of compressed sensing and parallel imaging can be applied to both spectrally adiabatic inversion recovery and the Dixon technique for fat suppression (Figs. 5, 7).

Non-Cartesian acquisition schemes, such as radial or spiral sampling, can be performed for dynamic imaging in combination with compressed sensing and parallel imaging. Stack-of-stars sampling, which uses radial acquisition (Fig. 1C), is the most common acquisition scheme for body imaging. It uses in-plane radial acquisition and through-plane Cartesian acquisition (24, 34). For the radial trajectory, a golden-angle ordering scheme is beneficial. With a suitable increment between angles of subsequent spokes, e.g. $112.2^{\circ}$, it can be guaranteed that k-space is almost uniformly sampled in almost arbitrary time intervals (35). In golden-angle radial sparse parallel (GRASP) imaging, a following spoke fills the largest gap between prior spokes, which results in uniform k-space coverage at any time during a scan $(23,24,36,37)$. Using the technique, high temporal resolution and high spatial resolution can be obtained for dynamic phases. In other
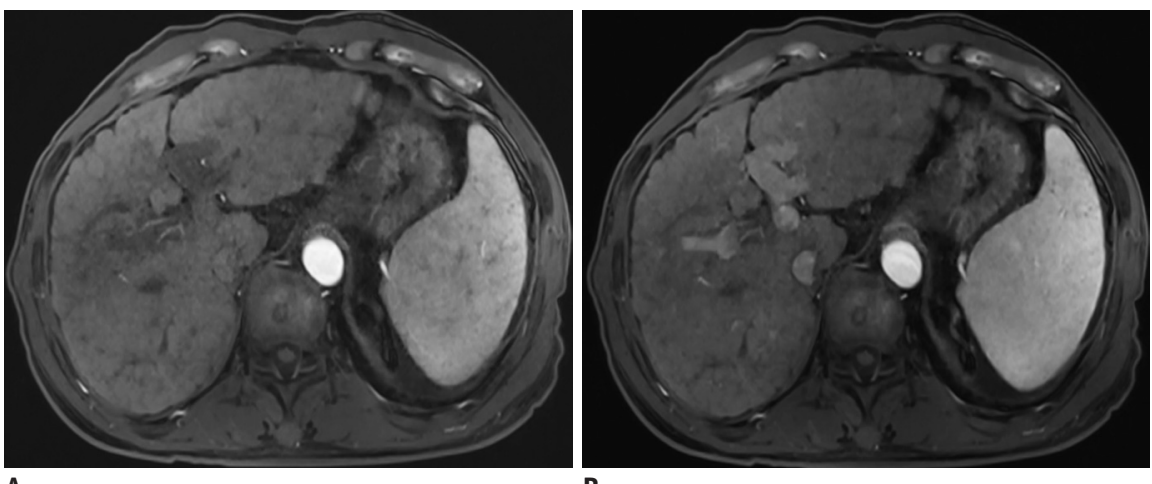

B

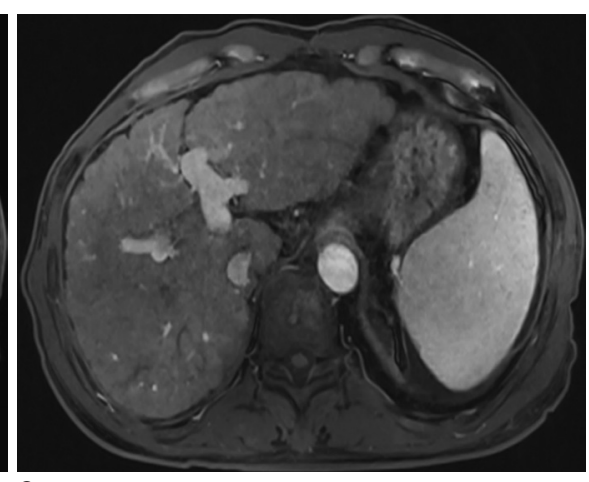

C

Fig. 5. Multi-arterial phase of gadoxetic acid-enhanced MRI in 63-year-old man obtained using compressed sensing and parallel imaging.

First (A), second (B), and third (C) arterial phases clearly captured different timings of contrast-enhancement of liver, with sufficient spatial resolution (reconstruction voxel size of $0.98 \times 1.41 \times 3 \mathrm{~mm}$ ), without noticeable temporal blurring in single breath-hold. Temporal resolution of each phase was 5.3 seconds.

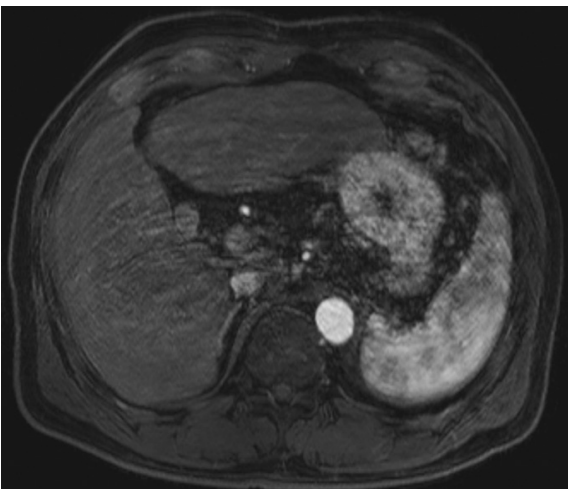

A

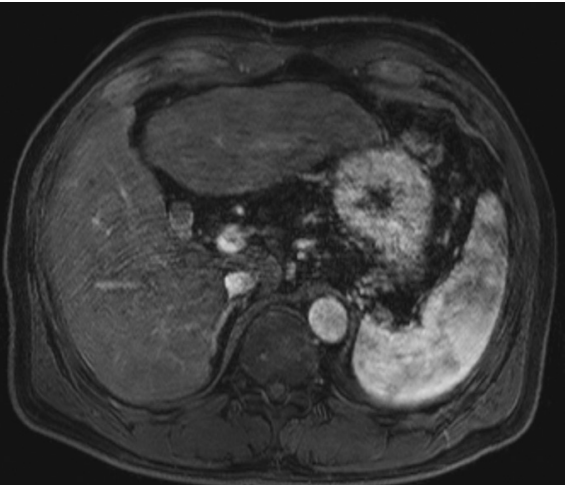

B

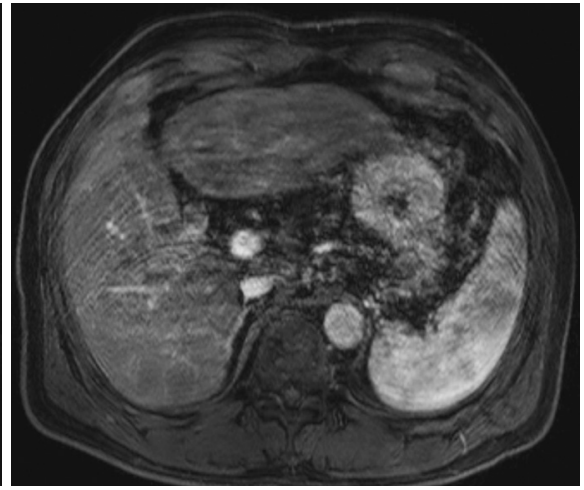

C

Fig. 6. Multi-arterial phase of gadoxetic acid-enhanced MRI in 66-year-old man obtained using view-sharing technique. All three arterial phases (A-C) show persistent motion artifacts, which decrease image quality. 

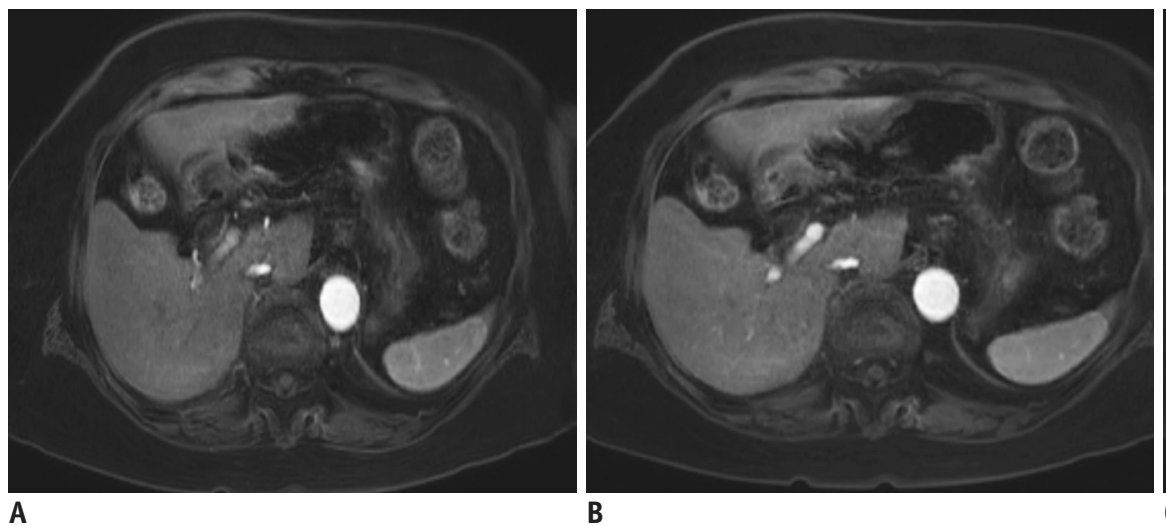

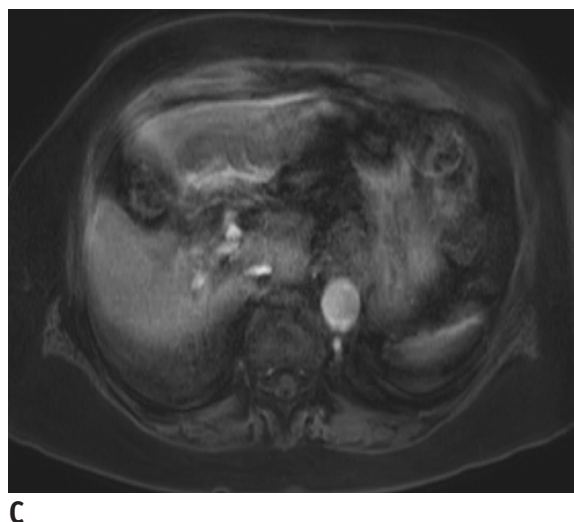

Fig. 7. Multi-arterial phase of gadoxetic acid-enhanced MRI in 88-year-old woman obtained using compressed sensing and parallel imaging.

Even though patient failed to hold her breath during scan, first (A) and second (B) phases were saved because motion artifact was limited to last phase (C).

words, images can be "retrospectively" reconstructed using subsets of data owing to the uniform coverage $(38,39)$. Using GRASP, images with variable temporal resolution can be reconstructed from a single examination $(36,38$, 39) (Fig. 8), and the highest temporal resolution has been reported as less than 3 seconds per volume for liver MRI (38). Although images with the highest temporal resolution (less than 3 seconds) are not desired in routine clinical practice, due to substantial artifacts, the achievable temporal resolution of GRASP is encouraging. In addition, the radial acquisition itself is motion-resistant as compared with Cartesian sampling, and data are often acquired under free-breathing conditions. The unique features of GRASP are further discussed in the following subsection.

\section{Free-Breathing T1-Weighted Images with Continuous Data Acquisition}

Although imaging with high temporal resolution can provide sufficient image quality in most patients, it does not eliminate the demand for breath-holding, nor address existing motion artifacts. Free-breathing images provide a potential solution to this issue. Free-breathing T1-weighted imaging can also be achieved using either Cartesian or nonCartesian acquisitions.

Free-breathing Cartesian acquisitions have been reported for dynamic imaging of liver MRI $(31,32)$. Variable density undersampling scheme is combined with parallel imaging and compressed sensing using a prototypical sequence (compressed-sensing volumetric interpolated breath-hold examination [CS-VIBE], Siemens Healthineers, Erlangen, Germany), with an 11-second temporal resolution (31, 32). Because the Cartesian acquisition is more sensitive to motion, motion correction is mandatory for achieving acceptable image quality. However, current respiratory triggering or gating techniques are not suitable for dynamic phase acquisition, due to scan inefficiency (40). For this sequence, the navigator signal is aligned with a preparation pulse and no temporal penalty is observed $(9,10)$. In combination with motion correction, free-breathing Cartesian undersampling provided fewer motion artifacts and better overall image quality than breath-hold Cartesian dynamic images in patients with limited breath-holding capacity or those at high risk of transient motion (Fig. 9) (31, 32).

Non-Cartesian acquisitions are often less sensitive to motion, which is an advantage for free-breathing imaging. GRASP, describe above, belongs to this category. However, substantial motion also creates motion artifacts in radial acquisitions, and motion correction would be helpful for improving image quality as compared with non-gated images (39).

For both free-breathing Cartesian and non-Cartesian sampling schemes, motion correction can be performed retrospectively (41). For Cartesian sampling, an implemented navigator signal can be used $(31,32)$. For GRASP, central k-space is sampled continuously and a self-gated signal (Fig. 10) can be extracted without additional navigator signals or respiratory bellows $(39,42)$. The simplest method of motion correction is "hard gating" in which an acceptance window is defined and the obtained motion signal is used to determine whether the data would be used or discarded for image reconstruction $(31,32,39)$. "Soft gating" is another option for reducing motion-related image blurring (43); it incorporates motion state weighting to penalize the motion state inconsistency. Motion-resolved reconstruction is a more 


\section{KJR}

advanced option in which motion state data is implemented during the image reconstruction process $(31,32,42,44)$. Because the reconstruction includes extra dimensions of the motion state, it is referred to as either eXtra-Dimension
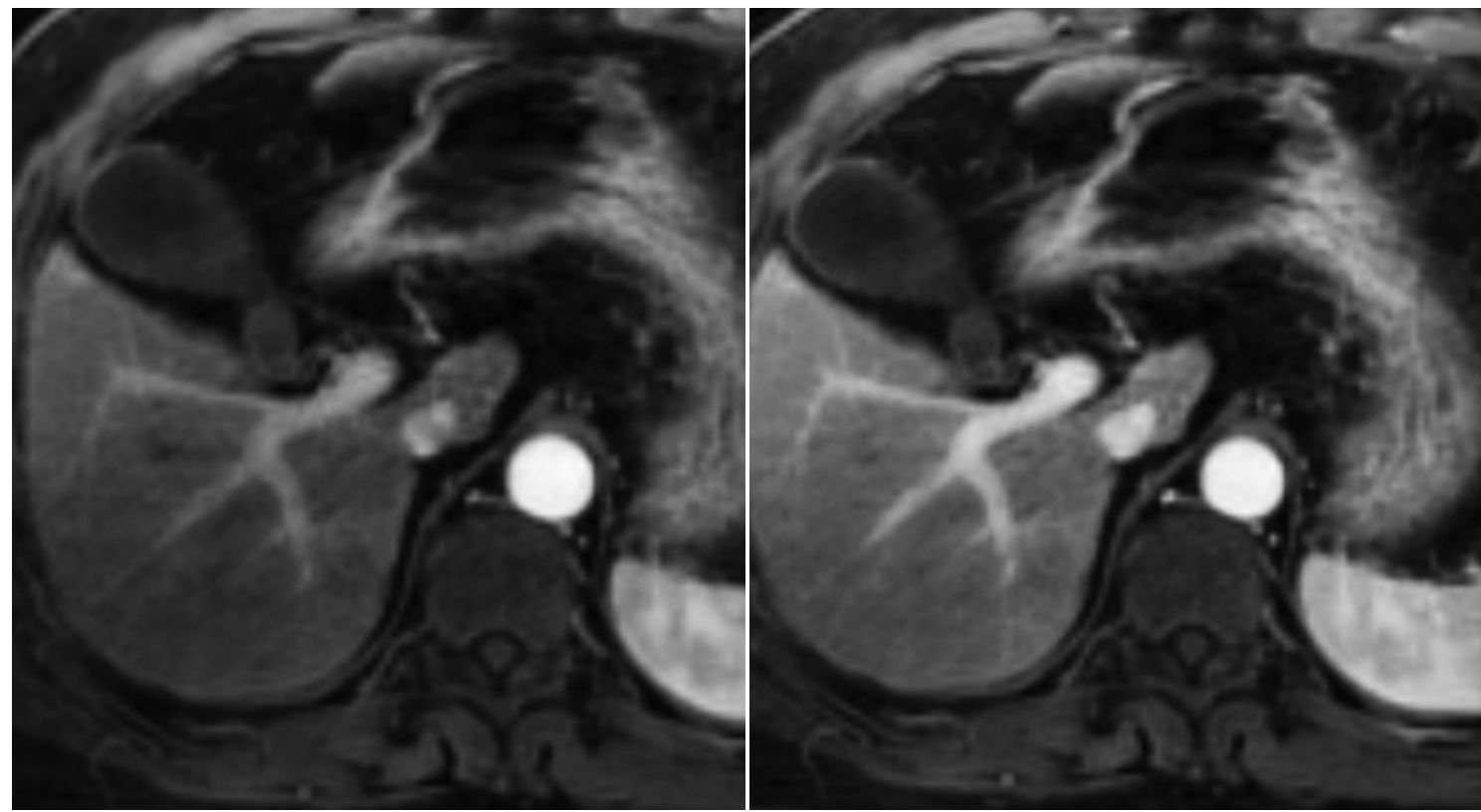

A

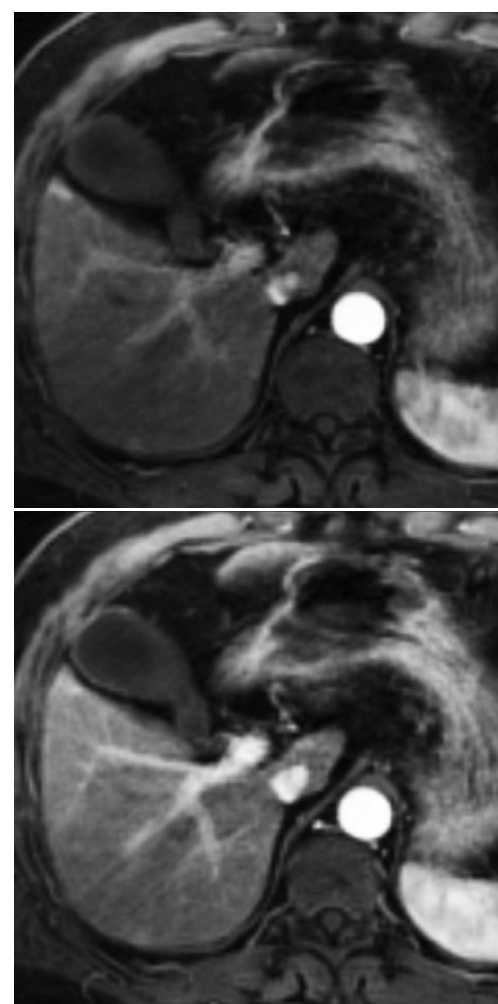

(XD)-GRASP or XD-VIBE (Fig. 11). Compared with hardgating, XD-VIBE showed better image quality due to further reduction of motion artifacts $(31,32)$.

Both free-breathing Cartesian and non-Cartesian 
sequences are clinically important in several ways. First, they reduce the need for patients to hold their breaths by providing motion robustness. This is presumably beneficial for patients with limited breath-holding capacity, noncooperative patients, including children, and patients at risk of transient motion after administration of contrast media. Even for patients with sufficient breath-holding capacity, it may enhance comfort by eliminating repeated breath-holding. Second, they provide a better workflow for radiology technicians by allowing continuous data acquisition (Fig. 12). Repeated instructions for optimal respiration would be omitted and arterial phase acquisition

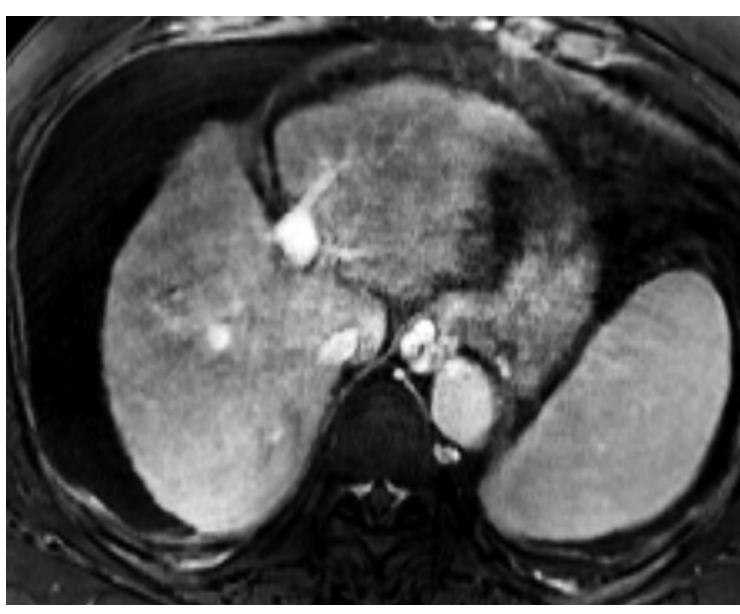

A

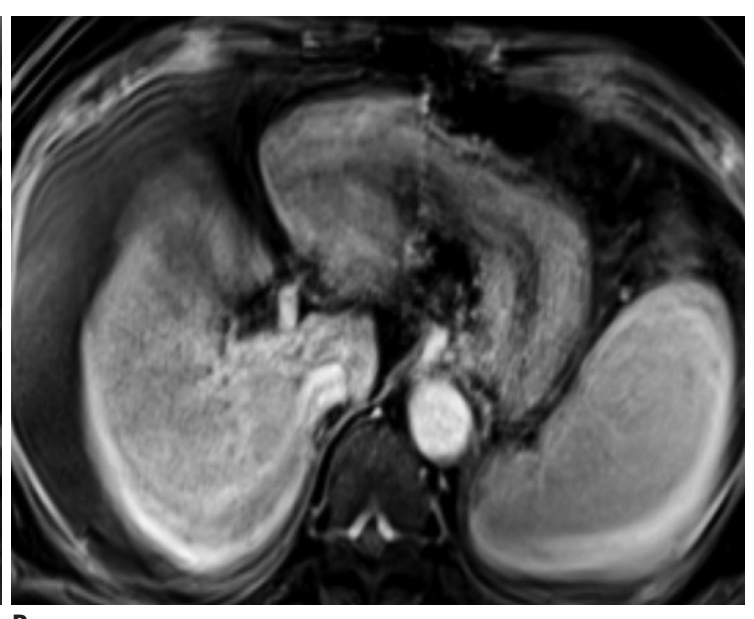

B

Fig. 9. T1-weighted images of gadoxetic acid-enhanced liver MRI in 56-year-old woman with limited breath-holding capacity. Motion artifacts are significantly less in free-breathing, motion-resolved reconstructed images (extra-dimension-VIBE, A) than in subsequent breath-hold 3D GRE transitional phase images (breath-hold VIBE, B). GRE = gradient-echo, VIBE = volumetric interpolated breath-hold examination, $3 \mathrm{D}=$ three-dimensional

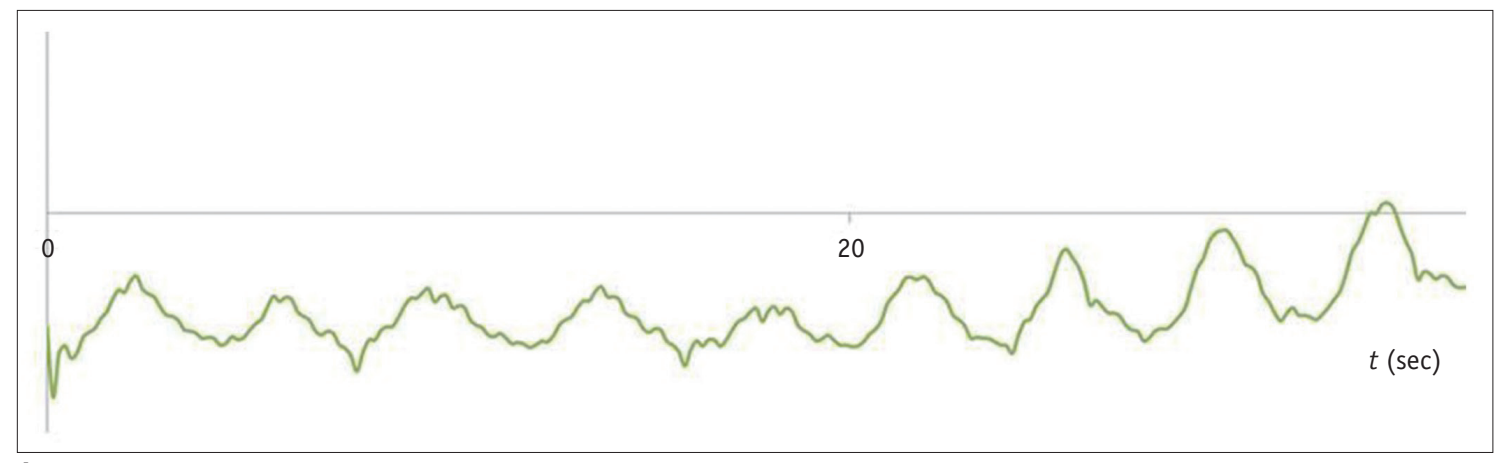

A

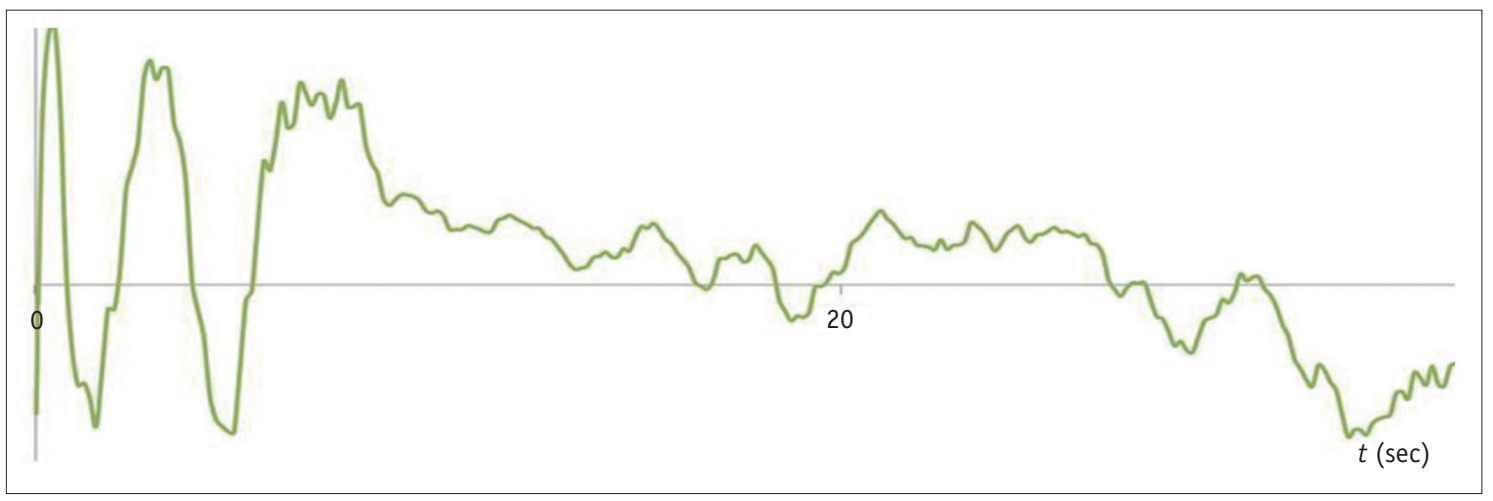

B

Fig. 10. Self-gated signals extracted from k-space in GRASP imaging sequence.

Regular breathing pattern (A) and irregular breathing pattern (B) are seen. 


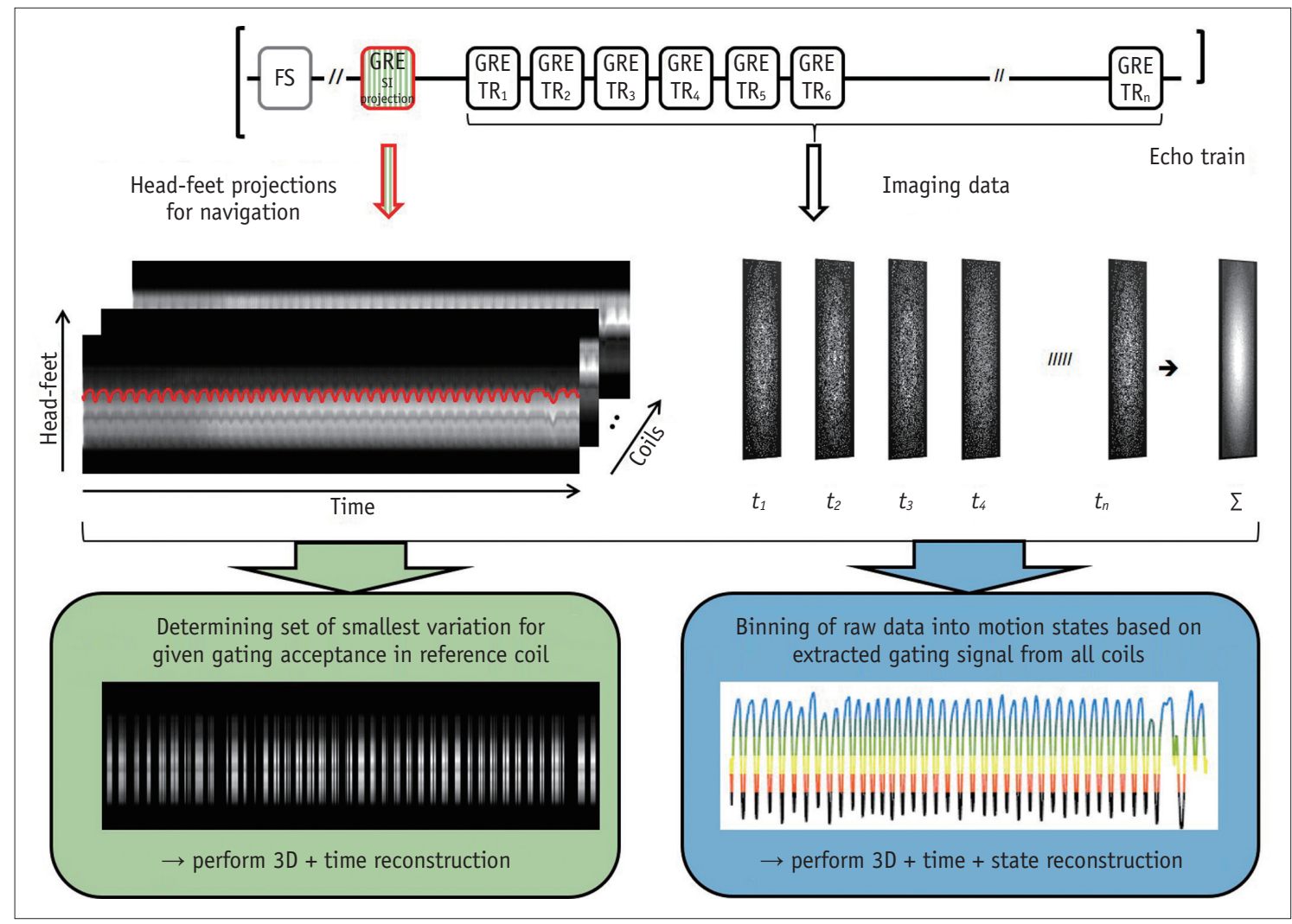

Fig. 11. Fast fat-saturated T1-weighted imaging acquires imaging data in form of echo trains following fat-suppression pulse. For free-breathing acquisitions, additional GRE with same excitation but with selectable readout direction can be inserted (top). Consequently, headfeet projections for each coil element can be obtained along with imaging data (middle). This can either be used for gated reconstruction that only utilizes specified fraction of data with smallest variation (bottom left) or for extracting gating signal to assign each echo train to motion state, followed by motion-resolved reconstruction (bottom right). FS = fat-suppressed, SI = signal intensity, TR = repetition time

using MR fluoroscopy may be unnecessary. Furthermore, they potentially reduce the need for re-examination of patients with motion during the acquisition. Currently, patients are referred for re-examination if motion artifacts cannot be corrected. Using the aforementioned sequences, retrospective motion correction is possible, and it provides clinically acceptable images in patients with motion during the scan. Third, GRASP provides a flexible temporal resolution from a single examination (Fig. 8). It makes it possible to recover a missing "arterial phase" without readministration of contrast media. Furthermore, the high temporal resolution images can capture critical hemodynamic information related to abdominal organs as well as tumors. Thus, current issues relating to dynamic imaging can be mitigated in coming years.

\section{D T1- and T2-Weighted Images with High Spatial Resolution}

Compared with CT or ultrasound, MRI has a lower spatial resolution. High spatial resolution images on MRI have been attempted using various techniques, and they have shown better lesion conspicuity $(45,46)$. However, there are inevitable drawbacks of aliasing artifacts and lowering the signal-to-noise ratio by increasing the acceleration factor of parallel imaging. By combining compressed sensing and parallel imaging, we may obtain images with high spatial resolution in an acceptable time frame. The aforementioned combination would reduce unfolding artifacts, which occur in images obtained with parallel imaging. In addition, the signal-to-noise penalty can be also decreased by combining parallel imaging and compressed sensing. By using compressed sensing, parallel imaging, and contrast media, MRI can achieve both high contrast resolution and high spatial resolution, which would be useful for detecting small lesions and anatomic structures (Fig. 13). Because compressed sensing can be combined with other sequences in addition to T1-weighted sequences, T2weighted imaging can also be acquired with better spatial resolution in an acceptable time frame by using compressed sensing (compressed SENSE, Philips Healthcare, Best, the 
Netherlands) (Fig. 14).

\section{D MRCP}

3D MRCP is one of the key sequences for evaluating the bile duct or pancreatic diseases. It is noninvasive, as compared with endoscopic retrograde cholangiopancreatography, and provides near isotropic volumetric data, as compared with 2D MRCP. Indeed, there

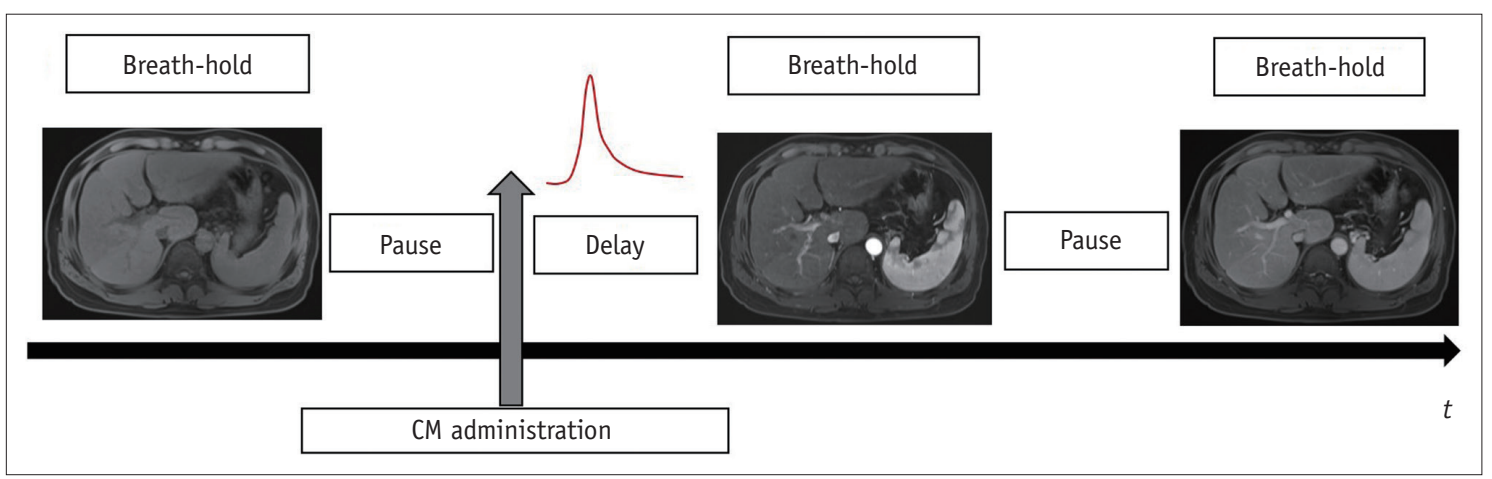

A

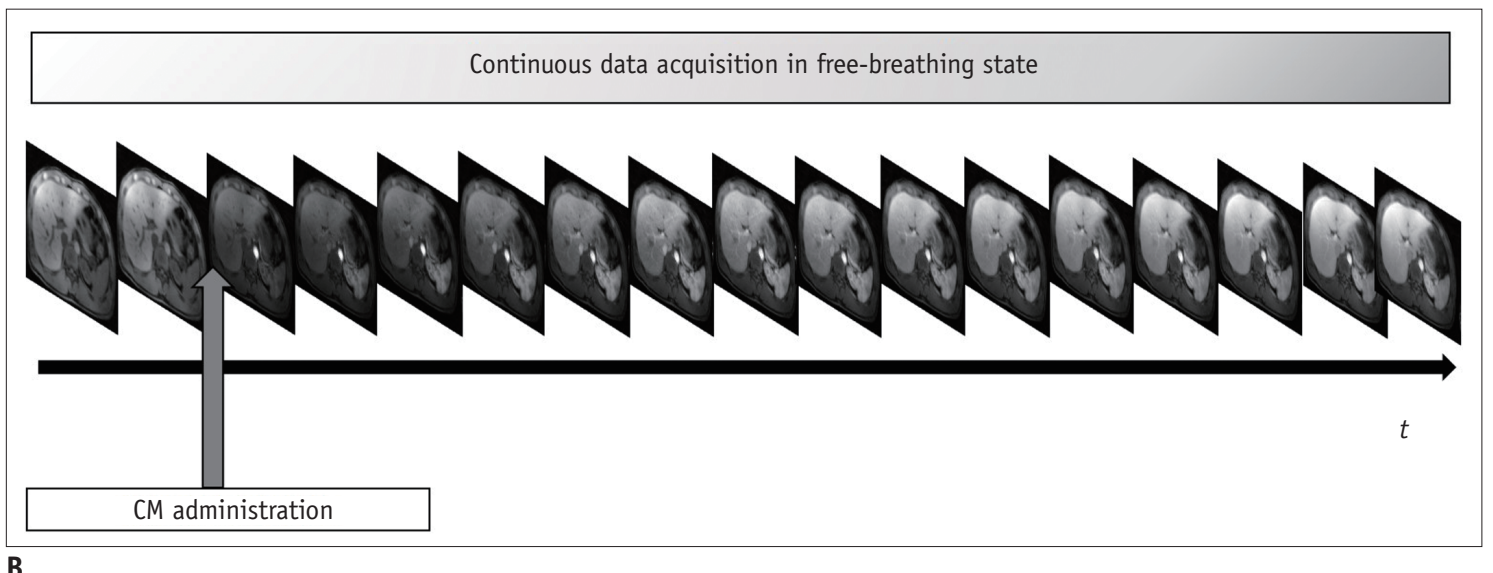

Fig. 12. Shift of acquisition scheme in contrast-enhanced abdominal MRI.

Current protocol of dynamic sequence $(\mathbf{A})$ includes several pauses and instances of breath-holding. In dynamic sequences using compressed sensing VIBE or GRASP, continuous data acquisition is possible (B) without breath-holding, because images are retrospectively reconstructed including motion correction. $\mathrm{CM}=$ contrast media

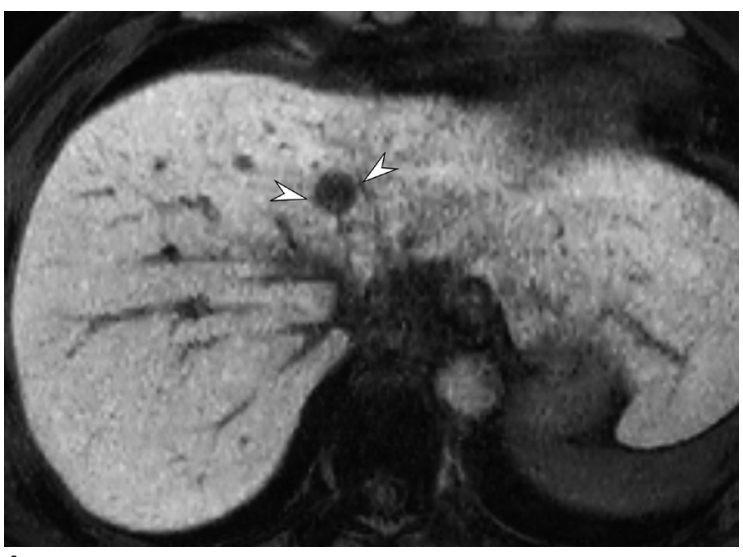

A

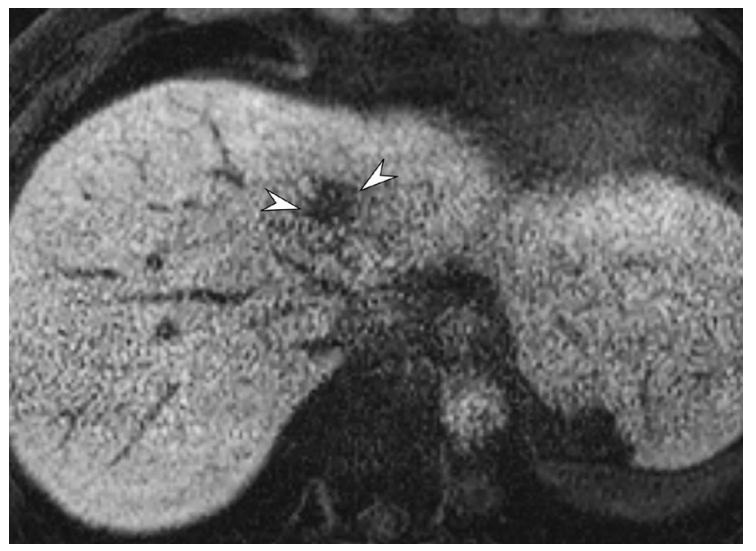

B

Fig. 13. Hepatobiliary phase of gadoxetic acid-enhanced liver MRI in 51-year-old man.

Image obtained with compressed sensing and parallel imaging $(\mathbf{A})$ shows less image noise and better overall image quality than that obtained with parallel imaging only (B). Treated hepatocellular carcinoma (arrowheads) is more visible in image obtained by using both compressed sensing and parallel imaging, than in that obtained with parallel imaging alone. Acquisition time is 15 seconds for both images and spatial resolution is same (reconstruction voxel size: $0.98 \times 0.98 \times 1.5 \mathrm{~mm}$ ). 


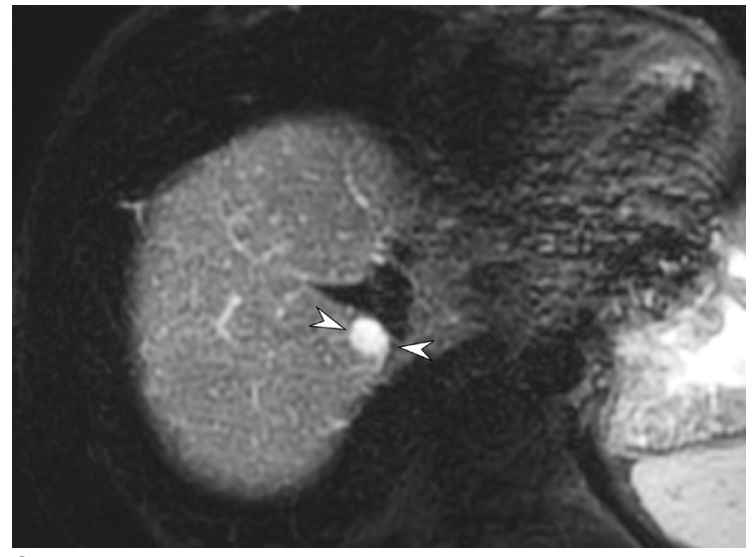

A

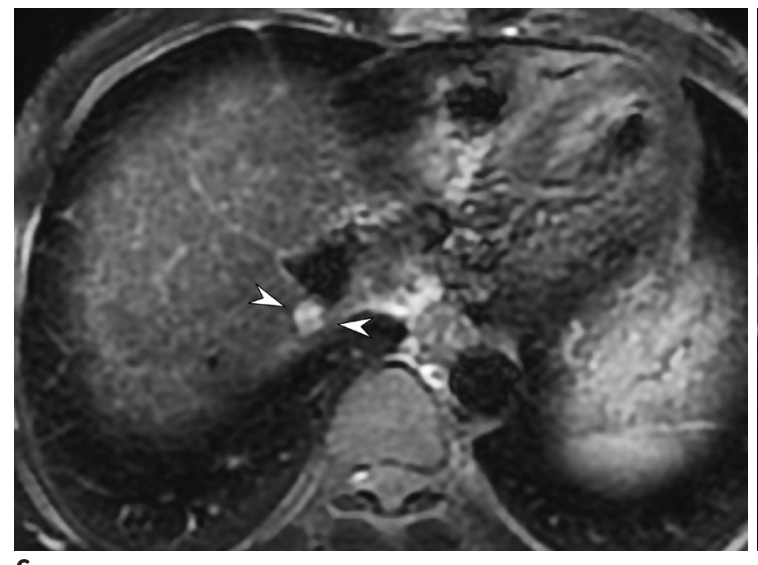

C

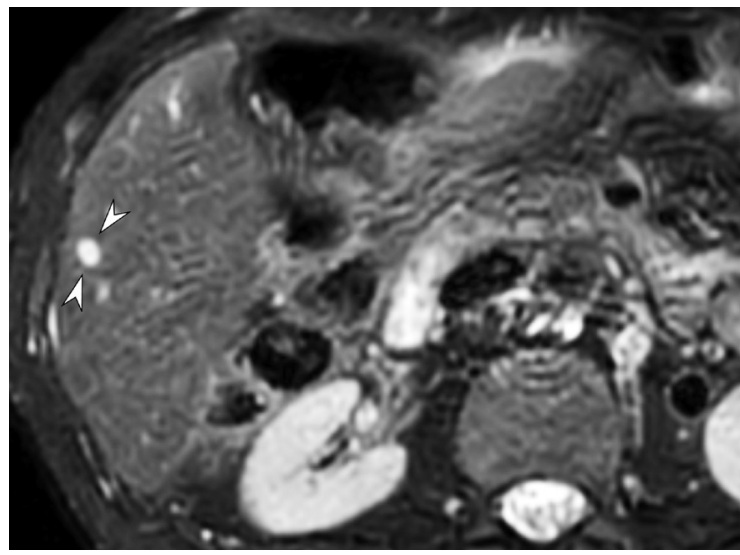

B

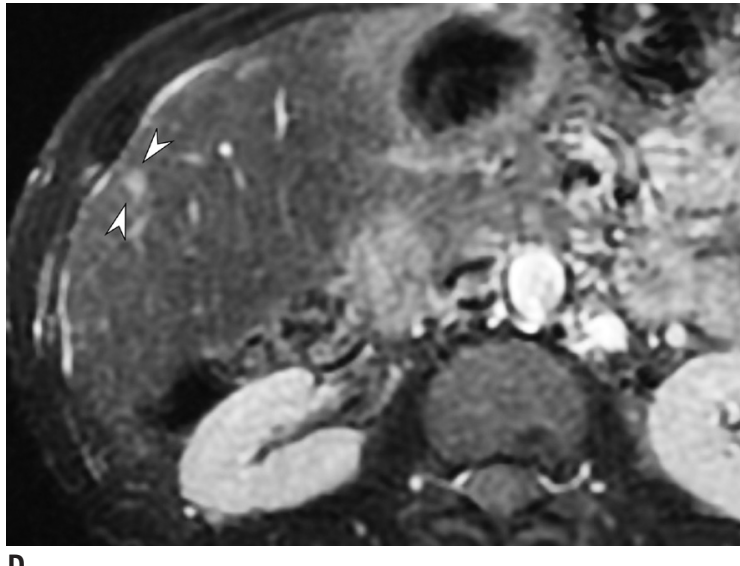

D

Fig. 14. T2-weighted image of 49-year-old man with hemangiomas.

2D T2-weighted images using compressed sensing and parallel imaging with 4-mm slice thickness and 4-mm gap (A, B) provide better conspicuity of small hemangiomas (arrowheads) than that obtained in 2D T2-weighted images with 8-mm slice thickness and 8-mm gap (C, D). TR/TE were $3240 / 80 \mathrm{~ms}$ (A, B) and 2050/83.6 ms (C, D), respectively. TE = echo time, 2D = two-dimensional

have been several reports that 3D MRCP showed better duct visibility or diagnostic performance than 2D MRCP (47-49). However, the acquisition time is long because respiratory triggering is routinely used to obtain volumetric data of a large field of view. Compressed sensing is an effective strategy for acquiring 3D MRCP because 3D MRCP is sparse in the image domain. In other words, 3D MRCP uses the high contrast of fluid-containing structures, such as the bile duct or pancreatic duct, and any other background signal is suppressed. Thus, it is easily anticipated that image reconstruction can be achieved from a small number of data samples, and therefore, that scan time can be reduced.

To reduce data sampling, variable-density, random undersampling has been used; this includes a variabledensity Poisson disk pattern (50-52) or a variable-density Gaussian incoherent sampling model $(53,54)$. In addition, either GRAPPA or SENSE can be applied to accelerate the scan speed, as well as to aid in the preservation of data consistency and reduction of the reconstruction time. As expected, the combination of compressed sensing and parallel imaging shortened the scan time of 3D MRCP in previous studies $(50,51,54)$. In those studies, applying compressed sensing and parallel imaging reduced acquisition time by $50 \%$. Specifically, respiratory-triggered 3D MRCP can be achieved within 2 or 3 minutes $(50,51$, 54). In addition, overall image quality was not significantly different from that of 3D MRCP using parallel imaging only (Fig. 15) $(50,51,54)$. This was an encouraging result in terms of improving MRI workflow and reducing the burden for radiologists, radiology technicians, and patients.

Regardless of these benefits, respiratory-triggered of 3D MRCP still has several issues. A scan time of 2 or 3 minutes is still lengthy as compared with thick-slab 2D MRCP. Moreover, image quality and acquisition time depend heavily on patients' respiratory pattern. In other words, the scan time is often unpredictable and image quality is often 


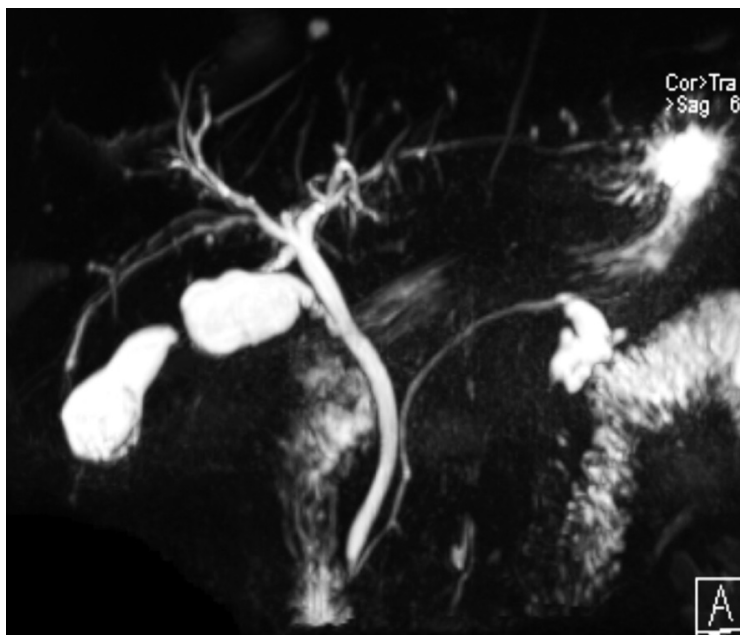

A

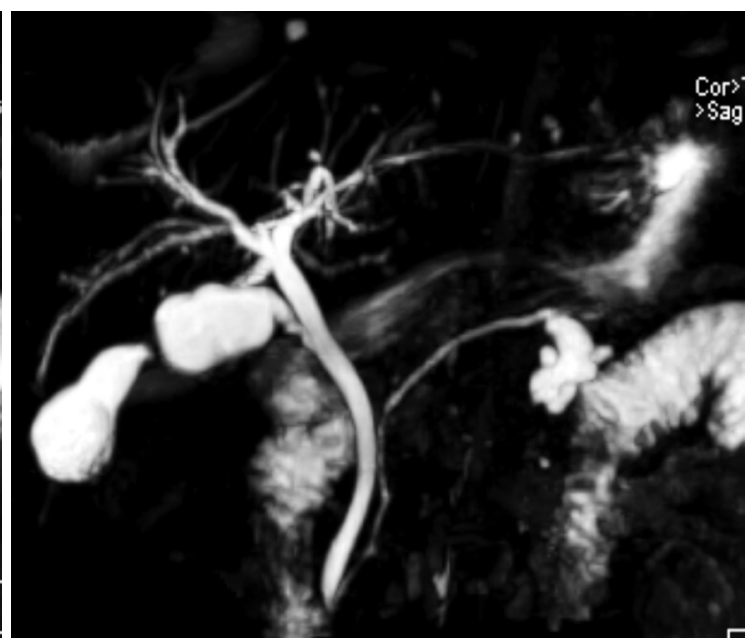

B

Fig. 15. Respiratory-triggered 3D MRCP in 67-year-old man.

Conventional 3D MRCP (A) and compressed sensing 3D MRCP (B) show comparable image quality, with acquisition times of 5 minutes 35 seconds and 2 minutes 4 seconds, respectively. TR/TE was 4172/702 ms for conventional 3D MRCP (A) and 3861/725 ms for compressed sensing 3D MRCP (B). $\mathrm{MRCP}=$ magnetic resonance cholangiopancreatography

unsatisfactory when patients have an irregular breathing rhythm. In those patients, respiratory-triggered 3D MRCP, even using both compressed sensing and parallel imaging, is unable to solve the problem.

Continuous efforts have been made to reduce scan time further. Now 3D MRCP can be acquired with a single breathhold by exploiting the capability of a combination of compressed sensing and parallel imaging (50-52). In these studies, only $4.5-5 \%$ of k-space data was sampled, resulting in a more than 10 times faster acquisition speed than that of conventional respiratory-triggered 3D MRCP sequences. The image quality was not significantly inferior to that of conventional respiratory-triggered 3D MRCP (52) and was even better in terms of blurring and motion artifacts (50, 51). Compared with respiratory-triggered 3D MRCP, breathhold 3D MRCP also reduced the incidence of undiagnostic scans or severe artifacts $(50,51)$. This is a remarkable advance in abdominal MRI and compressed sensing implementation in clinical practice. Even though breath-hold 3D MRCP has been attempted since its initial presentation, using techniques and sequences other than compressed sensing $(47,55)$, those techniques were not implemented in clinical practice due to the inconsistent and unsatisfactory image quality as compared with conventional respiratorytriggered 3D MRCP. However, compared with prior methods, the new breath-hold 3D MRCP using compressed sensing seems to provide consistent image quality and is useful in patients failing to breathe regularly (Fig. 16).

A recent study has suggested a modified protocol for breath-hold 3D MRCP with high resolution and a small field of view, using a large acceleration factor, oversampling, and saturation band (56). As expected, high-resolution breathhold 3D MRCP was better than the original breath-hold 3D MRCP in terms of image quality and peripheral bile duct and pancreatic duct visualization, resulting in a better depiction of pancreatic duct abnormality (56).

\section{Other Methods for Rapid Abdominal MRI}

\section{Simultaneous Multi-Slice DWI}

Although compressed sensing is a promising technique for accelerating the acquisition speed of MRI, its benefit seems to be limited in DWI. This is because the single-shot echoplanar imaging (EPI) scheme that is the most commonly used in DWI is highly effective, and there is insufficient room to achieve sparsity in 2D static images as compared with 3D or dynamic images. Simultaneous multi-slice DWI (SMS-DWI) would be an option for reducing the scan time for DWI. SMS imaging excites several slices simultaneously using a multiband pulse $(57,58)$ that is carefully designed with consideration of constraints from increased specific absorption rates $(59,60)$. Slices are separated using information about coil sensitivities from phased array coils, gradients, or radiofrequency encoding. Because phased array coils typically have limited encoding power in transverse abdominal protocols in the slice direction, slice shifting is applied to separate slices and to improve the geometric factor $(16,61)$. A combination of parallel imaging and SMS 

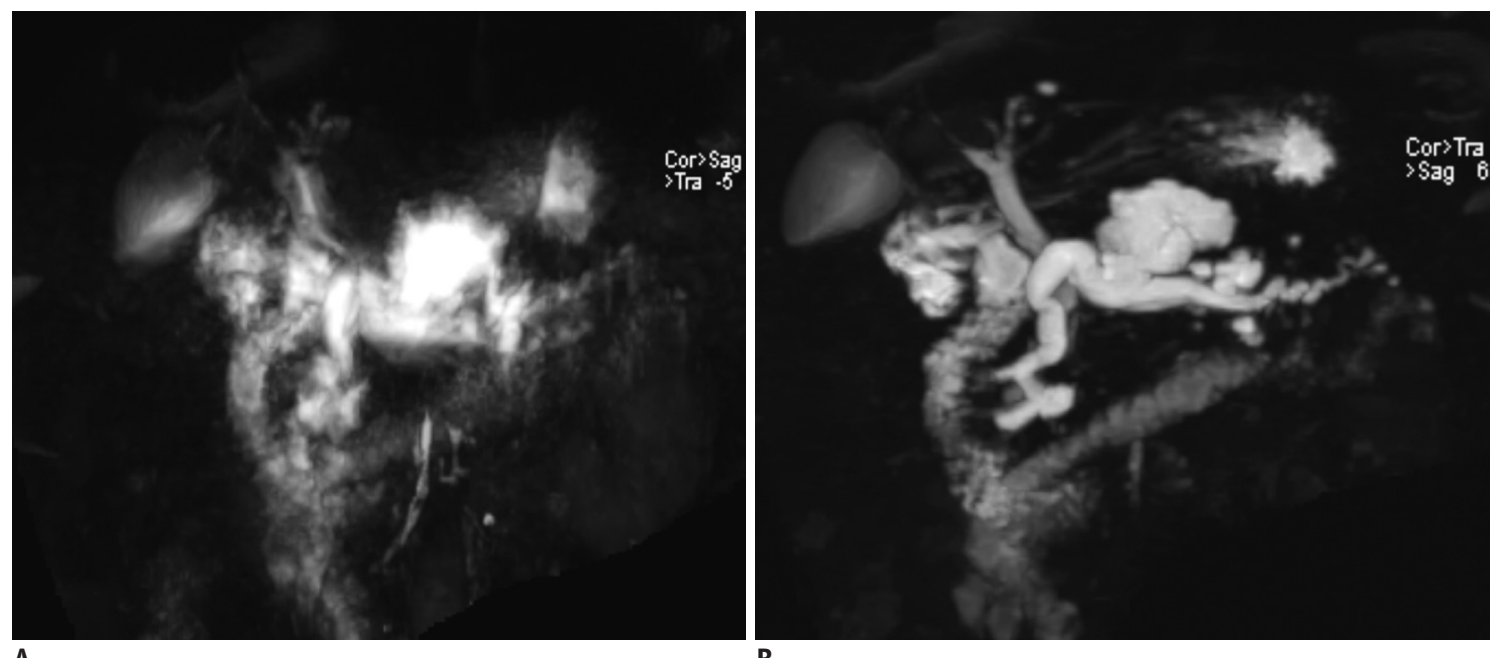

A B

Fig. 16. 3D MRCP using compressed sensing in 68-year-old woman.

Conventional respiratory-triggered 3D MRCP using parallel imaging (A) shows substantial motion artifacts due to irregular breathing patterns, whereas breath-hold 3D MRCP using compressed sensing and parallel imaging (B) shows acceptable image quality. TR/TE was $4421 / 699 \mathrm{~ms}$ for conventional respiratory-triggered 3D MRCP (A) and 1700/674 ms for breath-hold 3D MRCP (B).
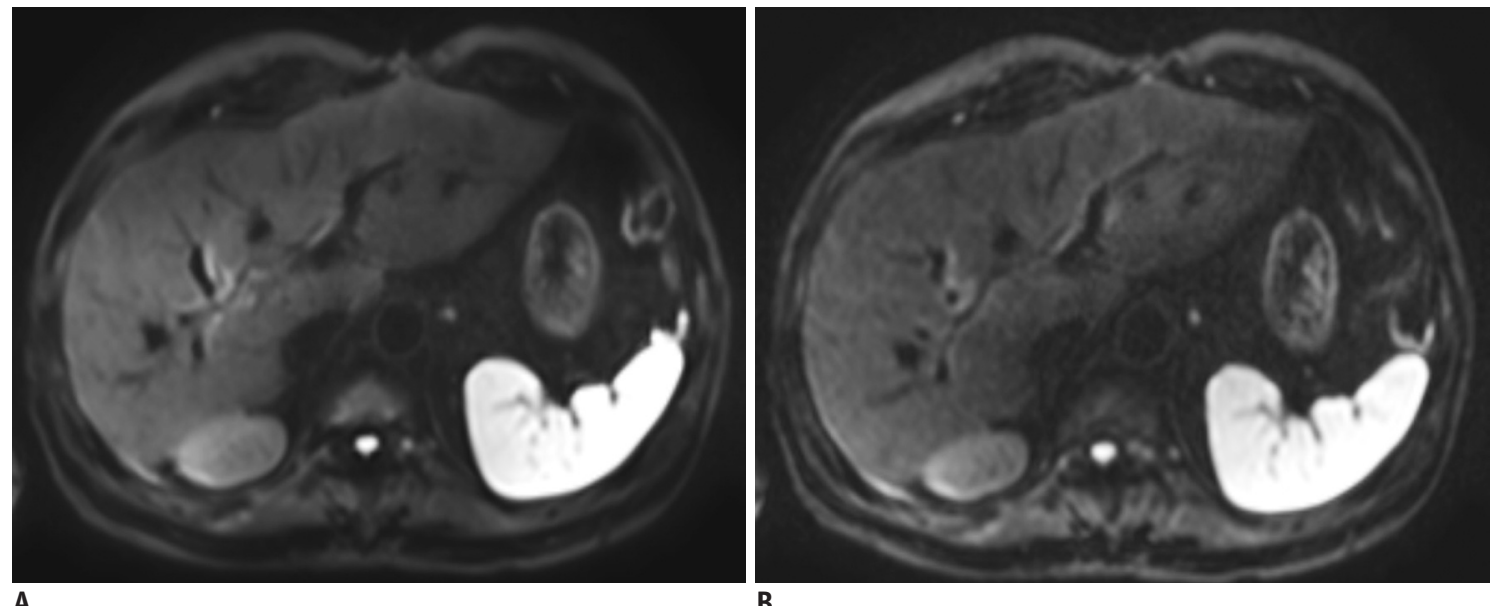

Fig. 17. Respiratory-triggered DWI using b-value of $800 \mathrm{~s} / \mathrm{mm}^{2}$ in 64 -year-old man.

Images of conventional DWI (A) and SMS-DWI (B) show comparable image quality, but scan time was significantly shorter in SMS-DWI than in conventional DWI. TR/TE were 2100/60 ms for conventional DWI (A) and 2200/62 ms for SMS-DWI (B). Field of view $\left(400 \times 320 \mathrm{~mm}^{2}\right)$ and matrix $(150 \times 120)$ were identical. DWI = diffusion-weighted imaging, SMS = simultaneous multi-slice

can create a synergistic effect for the following reasons. First, parallel imaging improves in-plane resolution and reduces echo train lengths. It can improve image quality by reducing blurring and distortion. SMS works in the slice direction, which significantly reduce scan time. Furthermore, SMS does not suffer from the inherent signal-to-noise ratio loss of undersampling acquisition (62).

It has been reported that SMS-DWI can provide acceptable image quality with a shorter acquisition time than conventional DWI (Fig. 17) (63-66). For abdominal protocols, SMS acceleration is now limited to a factor of 2 to ensure acceptable image quality $(66,67)$, because higher slice accelerations result in noise enhancement, related to increased g-factors, as well as more aliasing artifacts. This needs to be addressed in future. In addition, apparent diffusion coefficients should be compared between SMS and conventional DWI for quantitative measurements.

\section{Gradient and Spin-Echo for 3D MRCP}

Gradient and spin-echo (GRASE) is a combination of GRASE sequences. It uses a fast spin-echo or turbo spin-echo acquisition scheme and EPI readouts that follow the refocusing pulse of a fast spin-echo sequence (68). The acquisition speed can be accelerated by both the turbo factor of the 


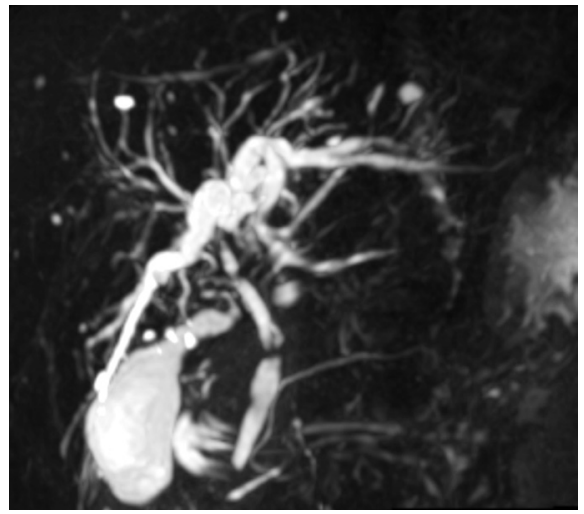

A

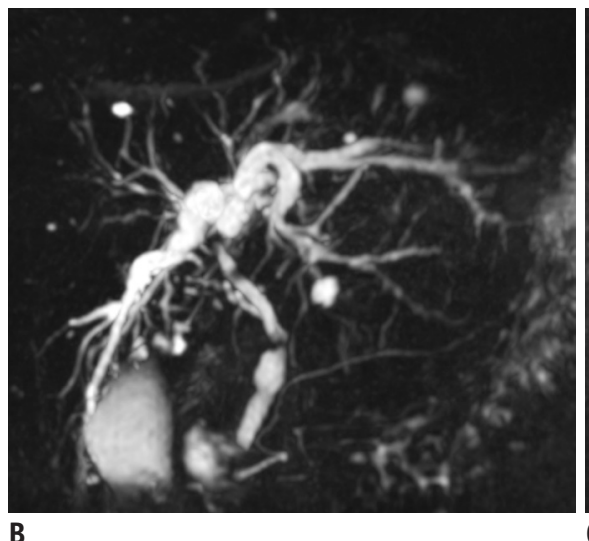

B

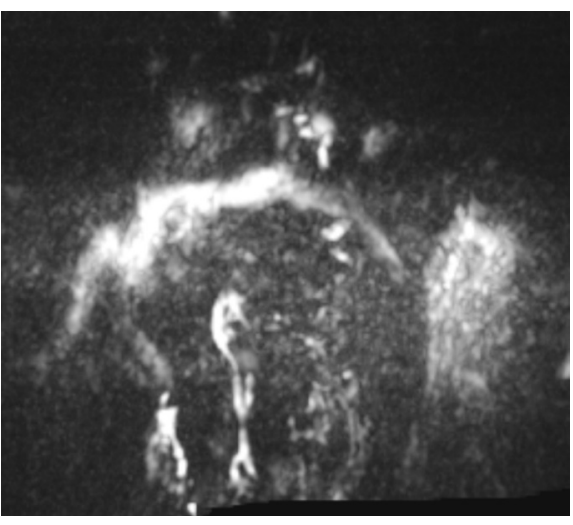

C

Fig. 18. 3D MRCP in 61-year-old woman with limited breath-holding capability.

Breath-hold 3D MRCP using gradient and spin-echo (A) shows comparable image quality to that of breath-hold 3D MRCP using compressed sensing (B), and better image quality than that of respiratory-triggered 3D MRCP (C).

turbo spin-echo sequence and the EPI factor of the gradientecho sequence $(68,69)$. Consequently, GRASE provides a lower acquisition time than a comparable turbo spin-echo sequence. In addition, the specific absorption rate is lower in GRASE, by using fewer radiofrequency pulses. Compared with EPI, GRASE has several advantages, including a better signalto-noise ratio and fewer susceptibility artifacts, owing to multiple refocusing pulses. Even though GRASE was developed in the early 1990s $(68,69)$, it has been selectively used for neuroimaging, until being applied for volumetric 3D MRCP $(70,71)$. This is because of its inherent limitation compared with turbo spin-echo sequences and a lack of parallel imaging. Although GRASE is less prone to susceptibility artifacts than EPI, it is more susceptible to field inhomogeneity than a turbo spin-echo sequence. In the case of old scanners, it would be challenging to achieve sufficient field homogeneity for $3 \mathrm{D}$ MRCP that covers a large field of view, resulting in inconsistent image quality in 3D MRCP using GRASE. Fortunately, recent scanners offer better field homogeneity and parallel imaging is available (70). Recent papers have reported that 3D MRCP can be achieved in a single breath-hold using a combination of GRASE and parallel imaging and that it provided reduced motion artifacts and better overall image quality than conventional respiratory-triggered 3D MRCP (Fig. 18) (70, 71). Although compressed sensing and parallel imaging techniques provide excellent results, 3D MRCP using GRASE would be an alternative option for scanners where compressed sensing is not available (Fig. 18).

\section{CONCLUSION}

Rapid MRI is crucial for abdominal imaging due to the critical limitation with regard to breath-holding. Compressed sensing has improved the temporal resolution and spatial resolution of abdominal MRI, when used in combination with parallel imaging. In addition, it allows continuous data acquisition in free-breathing for dynamic images in combination of other techniques and breath-hold volumetric data acquisition for 3D MRCP. Therefore, the compressed sensing technique is likely to change clinical practice in the coming years. Regarding dynamic images, a combination of compressed sensing and parallel imaging would minimize breath-holding duration or eliminate the need for holding the breath by simultaneously using a motion correction technique. In addition, free-breathing techniques can potentially correct motion artifacts retrospectively in MR images. As mentioned earlier, free-breathing, continuous data acquisition with retrospective motion correction would improve patients' comfort, in addition to reducing the workload of radiologists and radiology technicians, by reducing the necessity of re-examination, and enhancing overall image quality.

In available scanners, conventional respiratory-triggered 3D MRCP has already been replaced with compressed sensing respiratory-triggered 3D MRCP that reduces scan time by approximately $50 \%$. This will have a marked clinical impact, especially when breath-hold 3D MRCP is the first sequence performed; the scan time will then be reduced from 5-6 minutes to less than 20 seconds. Furthermore, compressed sensing respiratory-triggered 3D MRCP will be performed only in patients with limited breath-holding capacity. It will substantially improve the current workflow and image quality. Furthermore, SMS-DWI can reduce the scan time for DWI. 
The major limitation of compressed sensing in clinical practice would be the computational burden, leading a long reconstruction time. Although this can be ameliorated by using a graphics processing unit, advanced reconstruction, including extra dimension reconstruction, is still demanding. We expect that this issue will also be resolved by technical developments, including artificial intelligence for image reconstruction, in the coming years.

In summary, rapid MRI using compressed sensing, parallel imaging, and SMS acquisition have been implemented in clinical examinations. They have contributed to enhancing both the temporal and spatial resolution of abdominal MRI, optimizing the workflow, and improving patients' experience.

\section{Conflicts of Interest}

Two authors are employees of Siemens Healthcare (Nickel MD) and Philips Healthcare (Peeters JM).

\section{Acknowledgments}

We thank to Benjamin Latimer, MA, for his editorial assistance and Berthold Kiefer, Ph.D., for the helpful discussion.

\section{ORCID iD}

Jeong Hee Yoon

https://orcid.org/0000-0002-9925-9973

Jeong Min Lee

https://orcid.org/0000-0003-0561-8777

\section{REFERENCES}

1. Bashir MR, Castelli P, Davenport MS, Larson D, Marin D, Hussain HK, et al. Respiratory motion artifact affecting hepatic arterial phase MR imaging with gadoxetate disodium is more common in patients with a prior episode of arterial phase motion associated with gadoxetate disodium. Radiology 2015;274:141-148

2. Pietryga JA, Burke LM, Marin D, Jaffe TA, Bashir MR. Respiratory motion artifact affecting hepatic arterial phase imaging with gadoxetate disodium: examination recovery with a multiple arterial phase acquisition. Radiology 2014;271:426434

3. Griswold MA, Jakob PM, Heidemann RM, Nittka M, Jellus V, Wang J, et al. Generalized autocalibrating partially parallel acquisitions (GRAPPA). Magn Reson Med 2002;47:1202-1210

4. Pruessmann KP, Weiger M, Scheidegger MB, Boesiger P. SENSE: sensitivity encoding for fast MRI. Magn Reson Med 1999;42:952-962
5. Hoogeveen RM, von Falkenhausen M, Gieseke J. Fast dynamic, high resolution contrast-enhanced MR angiography with CENTRA keyhole and SENSE. Proceedings of the 12th scientific meeting and exhibition of the International Society for Magnetic Resonance in Medicine;2004 May 15-21;Kyoto, Japan

6. Kazmierczak PM, Theisen D, Thierfelder KM, Sommer WH, Reiser MF, Notohamiprodjo M, et al. Improved detection of hypervascular liver lesions with CAIPIRINHA-Dixon-TWISTvolume-interpolated breath-hold examination. Invest Radiol 2015;50:153-160

7. van Vaals JJ, Brummer ME, Dixon WT, Tuithof HH, Engels $H$, Nelson RC, et al. "Keyhole" method for accelerating imaging of contrast agent uptake. J Magn Reson Imaging 1993;3:671675

8. Willinek WA, Hadizadeh DR, von Falkenhausen M, Urbach $H$, Hoogeveen R, Schild HH, et al. 4D time-resolved MR angiography with keyhole (4D-TRAK): more than 60 times accelerated MRA using a combination of CENTRA, keyhole, and SENSE at 3.0T. J Magn Reson Imaging 2008;27:1455-1460

9. Yoon JH, Lee JM, Yu MH, Kim EJ, Han JK. Triple arterial phase MR imaging with gadoxetic acid using a combination of contrast enhanced time robust angiography, keyhole, and viewsharing techniques and two-dimensional parallel imaging in comparison with conventional single arterial phase. Korean J Radiol 2016;17:522-532

10. Hope TA, Saranathan M, Petkovska I, Hargreaves BA, Herfkens RJ, Vasanawala SS. Improvement of gadoxetate arterial phase capture with a high spatio-temporal resolution multiphase three-dimensional SPGR-Dixon sequence. J Magn Reson Imaging 2013;38:938-945

11. Saranathan M, Rettmann DW, Hargreaves BA, Clarke SE, Vasanawala SS. DIfferential subsampling with Cartesian ordering (DISCO): a high spatio-temporal resolution Dixon imaging sequence for multiphasic contrast enhanced abdominal imaging. J Magn Reson Imaging 2012;35:14841492

12. Donoho DL. Compressed sensing. IEEE T Inform Theory 2006;52:1289-1306

13. Lustig M, Donoho D, Pauly JM. Sparse MRI: the application of compressed sensing for rapid MR imaging. Magn Reson Med 2007;58:1182-1195

14. Lustig M, Donoho DL, Santos JM, Pauly JM. Compressed sensing MRI. IEEE Signal Process Mag 2008;25:72-82

15. Peeters JM, Fuderer M. SENSE with improved tolerance to inaccuracies in coil sensitivity maps. Magn Reson Med 2013;69:1665-1669

16. Breuer FA, Blaimer M, Mueller MF, Seiberlich N, Heidemann RM, Griswold MA, et al. Controlled aliasing in volumetric parallel imaging (2D CAIPIRINHA). Magn Reson Med 2006;55:549-556

17. Yu MH, Lee JM, Yoon JH, Kiefer B, Han JK, Choi BI. Clinical application of controlled aliasing in parallel imaging results in a higher acceleration (CAIPIRINHA)-volumetric interpolated breathhold (VIBE) sequence for gadoxetic acid-enhanced liver 
MR imaging. J Magn Reson Imaging 2013;38:1020-1026

18. Feng L, Benkert T, Block KT, Sodickson DK, Otazo R, Chandarana H. Compressed sensing for body MRI. J Magn Reson Imaging 2017;45:966-987

19. Cook RL. Stochastic sampling in computer graphics. ACM TOG 1986;5:51-72

20. Kim D, Dyvorne HA, Otazo R, Feng L, Sodickson DK, Lee VS. Accelerated phase-contrast cine MRI using k-t SPARSE-SENSE. Magn Reson Med 2012;67:1054-1064

21. Paul J, Wundrak S, Bernhardt P, Rottbauer W, Neumann H, Rasche V. Self-gated tissue phase mapping using golden angle radial sparse SENSE. Magn Reson Med 2016;75:789-800

22. Murphy M, Alley M, Demmel J, Keutzer K, Vasanawala S, Lustig M. Fast $\ell 1-S P I R i T$ compressed sensing parallel imaging MRI: scalable parallel implementation and clinically feasible runtime. IEEE Trans Med Imaging 2012;31:1250-1262

23. Chandarana H, Feng L, Block TK, Rosenkrantz AB, Lim RP, Babb JS, et al. Free-breathing contrast-enhanced multiphase MRI of the liver using a combination of compressed sensing, parallel imaging, and golden-angle radial sampling. Invest Radiol 2013;48:10-16

24. Feng L, Grimm R, Block KT, Chandarana H, Kim S, Xu J, et al. Golden-angle radial sparse parallel MRI: combination of compressed sensing, parallel imaging, and golden-angle radial sampling for fast and flexible dynamic volumetric MRI. Magn Reson Med 2014;72:707-717

25. Otazo R, Kim D, Axel L, Sodickson DK. Combination of compressed sensing and parallel imaging for highly accelerated first-pass cardiac perfusion MRI. Magn Reson Med 2010;64:767-776

26. Davenport MS, Viglianti BL, Al-Hawary MM, Caoili EM, Kaza RK, Liu PS, et al. Comparison of acute transient dyspnea after intravenous administration of gadoxetate disodium and gadobenate dimeglumine: effect on arterial phase image quality. Radiology 2013;266:452-461

27. Motosugi U, Bannas P, Bookwalter CA, Sano K, Reeder SB. An investigation of transient severe motion related to gadoxetic acid-enhanced MR imaging. Radiology 2016;279:93-102

28. Park YS, Lee CH, Kim JW, Lee YS, Paek M, Kim KA. Application of high-speed $\mathrm{T} 1$ sequences for high-quality hepatic arterial phase magnetic resonance imaging: intraindividual comparison of single and multiple arterial phases. Invest Radiol 2017;52:605-611

29. Nam JG, Lee JM, Lee SM, Kang HJ, Lee ES, Hur BY, et al. High acceleration three-dimensional T1-weighted dual echo Dixon hepatobiliary phase imaging using compressed sensingsensitivity encoding: comparison of image quality and solid lesion detectability with the standard T1-weighted sequence. Korean J Radiol 2019;20:438-448

30. Vasanawala S, Murphy M, Alley M, Lai P, Keutzer K, Pauly J, et al. Practical parallel imaging compressed sensing MRI: summary of two years of experience in accelerating body MRI of pediatric patients. Proc IEEE Int Symp Biomed Imaging 2011;2011:1039-1043
31. Yoon JH, Yu MH, Chang W, Park JY, Nickel MD, Son Y, et al. Clinical feasibility of free-breathing dynamic T1-weighted imaging with gadoxetic acid-enhanced liver magnetic resonance imaging using a combination of variable density sampling and compressed sensing. Invest Radiol 2017;52:596604

32. Kaltenbach B, Bucher AM, Wichmann JL, Nickel D, Polkowski $C$, Hammerstingl R, et al. Dynamic liver magnetic resonance imaging in free-breathing: feasibility of a Cartesian T1weighted acquisition technique with compressed sensing and additional self-navigation signal for hard-gated and motionresolved reconstruction. Invest Radiol 2017;52:708-714

33. Levine E, Daniel B, Vasanawala S, Hargreaves B, Saranathan M. 3D Cartesian MRI with compressed sensing and variable view sharing using complementary poisson-disc sampling. Magn Reson Med 2017;77:1774-1785

34. Block KT, Chandarana H, Milla S, Bruno M, Mulholland T, Fatterpekar G, et al. Towards routine clinical use of radial stack-of-stars 3D gradient-echo sequences for reducing motion sensitivity. J Korean Soc Magn Reson Med 2014;18:87106

35. Winkelmann S, Schaeffter T, Koehler T, Eggers H, Doessel 0. An optimal radial profile order based on the golden ratio for time-resolved MRI. IEEE Trans Med Imaging 2007;26:68-76

36. Chandarana H, Block KT, Winfeld MJ, Lala SV, Mazori D, Giuffrida $E$, et al. Free-breathing contrast-enhanced T1weighted gradient-echo imaging with radial k-space sampling for paediatric abdominopelvic MRI. Eur Radiol 2014;24:320326

37. Chandarana H, Block TK, Rosenkrantz AB, Lim RP, Kim D, Mossa DJ, et al. Free-breathing radial 3D fat-suppressed T1-weighted gradient echo sequence: a viable alternative for contrast-enhanced liver imaging in patients unable to suspend respiration. Invest Radiol 2011;46:648-653

38. Chandarana H, Block TK, Ream J, Mikheev A, Sigal SH, Otazo $\mathrm{R}$, et al. Estimating liver perfusion from free-breathing continuously acquired dynamic gadolinium-ethoxybenzyldiethylenetriamine pentaacetic acid-enhanced acquisition with compressed sensing reconstruction. Invest Radiol 2015;50:88-94

39. Yoon JH, Lee JM, Yu MH, Hur BY, Grimm R, Block KT, et al. Evaluation of transient motion during gadoxetic acidenhanced multiphasic liver magnetic resonance imaging using free-breathing golden-angle radial sparse parallel magnetic resonance imaging. Invest Radiol 2018;53:52-61

40. Yoon JH, Lee JM, Lee ES, Baek J, Lee S, Iwadate Y, et al. Navigated three-dimensional T1-weighted gradient-echo sequence for gadoxetic acid liver magnetic resonance imaging in patients with limited breath-holding capacity. Abdom Imaging 2015;40:278-288

41. Lee CK, Seo N, Kim B, Huh J, Kim JK, Lee SS, et al. The effects of breathing motion on DCE-MRI images: phantom studies simulating respiratory motion to compare CAIPIRINHA-VIBE, radial-VIBE, and conventional VIBE. Korean 
J Radiol 2017;18:289-298

42. Chandarana H, Feng L, Ream J, Wang A, Babb JS, Block $\mathrm{KT}$, et al. Respiratory motion-resolved compressed sensing reconstruction of free-breathing radial acquisition for dynamic liver magnetic resonance imaging. Invest Radiol 2015;50:749756

43. Zhang T, Cheng JY, Potnick AG, Barth RA, Alley MT, Uecker $M$, et al. Fast pediatric 3D free-breathing abdominal dynamic contrast enhanced MRI with high spatiotemporal resolution. J Magn Reson Imaging 2015;41:460-473

44. Kim YC. Advanced methods in dynamic contrast enhanced arterial phase imaging of the liver. Investig Magn Reson Imaging 2019;23:1-16

45. Yoon JH, Lee JM, Yu MH, Kim EJ, Han JK, Choi BI. Highresolution T1-weighted gradient echo imaging for liver MRI using parallel imaging at high-acceleration factors. Abdom Imaging 2014;39:711-721

46. Yoon JH, Lee JM, Yu MH, Kim EJ, Han JK, Choi BI. Fatsuppressed, three-dimensional T1-weighted imaging using high-acceleration parallel acquisition and a dual-echo Dixon technique for gadoxetic acid-enhanced liver MRI at 3 T. Acta Radiol 2015;56:1454-1462

47. Sodickson A, Mortele KJ, Barish MA, Zou KH, Thibodeau S, Tempany CM. Three-dimensional fast-recovery fast spin-echo MRCP: comparison with two-dimensional single-shot fast spin-echo techniques. Radiology 2006;238:549-559

48. Yoon LS, Catalano OA, Fritz S, Ferrone CR, Hahn PF, Sahani DV. Another dimension in magnetic resonance cholangiopancreatography: comparison of 2- and 3-dimensional magnetic resonance cholangiopancreatography for the evaluation of intraductal papillary mucinous neoplasm of the pancreas. J Comput Assist Tomogr 2009;33:363-368

49. Kim JH, Hong SS, Eun HW, Han JK, Choi BI. Clinical usefulness of free-breathing navigator-triggered 3D MRCP in non-cooperative patients: comparison with conventional breath-hold 2D MRCP. Eur J Radiol 2012;81:e513-e518

50. Yoon JH, Lee SM, Kang HJ, Weiland E, Raithel E, Son Y, et al. Clinical feasibility of 3-dimensional magnetic resonance cholangiopancreatography using compressed sensing: comparison of image quality and diagnostic performance. Invest Radiol 2017;52:612-619

51. Zhu L, Wu X, Sun Z, Jin Z, Weiland E, Raithel E, et al. Compressed-sensing accelerated 3-dimensional magnetic resonance cholangiopancreatography: application in suspected pancreatic diseases. Invest Radiol 2018;53:150-157

52. Chandarana H, Doshi AM, Shanbhogue A, Babb JS, Bruno MT, Zhao $T$, et al. Three-dimensional MR cholangiopancreatography in a breath hold with sparsity-based reconstruction of highly undersampled data. Radiology 2016;280:585-594

53. Furlan A, Bayram E, Thangasamy S, Barley D, Dasyam A. Application of compressed sensing to $3 \mathrm{D}$ magnetic resonance cholangiopancreatography for the evaluation of pancreatic cystic lesions. Magn Reson Imaging 2018;52:131-136

54. Seo N, Park MS, Han K, Kim D, King KF, Choi JY, et al.
Feasibility of 3D navigator-triggered magnetic resonance cholangiopancreatography with combined parallel imaging and compressed sensing reconstruction at 3T. J Magn Reson Imaging 2017;46:1289-1297

55. Wielopolski PA, Gaa J, Wielopolski DR, Oudkerk M. Breathhold MR cholangiopancreatography with three-dimensional, segmented, echo-planar imaging and volume rendering. Radiology 1999;210:247-252

56. Zhu L, Xue H, Sun Z, Qian T, Weiland E, Kuehn B, et al. Modified breath-hold compressed-sensing 3D MR cholangiopancreatography with a small field-of-view and high resolution acquisition: clinical feasibility in biliary and pancreatic disorders. J Magn Reson Imaging 2018;48:13891399

57. Barth M, Breuer F, Koopmans PJ, Norris DG, Poser BA. Simultaneous multislice (SMS) imaging techniques. Magn Reson Med 2016;75:63-81

58. Müller S. Multifrequency selective rf pulses for multislice MR imaging. Magn Reson Med 1988;6:364-371

59. Setsompop K, Cohen-Adad J, Gagoski BA, Raij T, Yendiki A, Keil $B$, et al. Improving diffusion MRI using simultaneous multi-slice echo planar imaging. Neuroimage 2012;63:569580

60. Norris DG, Boyacioğlu R, Schulz J, Barth M, Koopmans PJ. Application of PINS radiofrequency pulses to reduce power deposition in RARE/turbo spin echo imaging of the human head. Magn Reson Med 2014;71:44-49

61. Breuer FA, Blaimer M, Heidemann RM, Mueller MF, Griswold MA, Jakob PM. Controlled aliasing in parallel imaging results in higher acceleration (CAIPIRINHA) for multi-slice imaging. Magn Reson Med 2005;53:684-691

62. Larkman DJ, Hajnal JV, Herlihy AH, Coutts GA, Young IR, Ehnholm G. Use of multicoil arrays for separation of signal from multiple slices simultaneously excited. J Magn Reson Imaging 2001;13:313-317

63. Taron J, Martirosian P, Erb M, Kuestner T, Schwenzer NF, Schmidt $H$, et al. Simultaneous multislice diffusion-weighted MRI of the liver: analysis of different breathing schemes in comparison to standard sequences. J Magn Reson Imaging 2016;44:865-879

64. Taron J, Martirosian P, Schwenzer NF, Erb M, Kuestner T, Weiß J, et al. Scan time minimization in hepatic diffusionweighted imaging: evaluation of the simultaneous multislice acceleration technique with different acceleration factors and gradient preparation schemes. MAGMA 2016;29:739-749

65. Obele CC, Glielmi C, Ream J, Doshi A, Campbell N, Zhang HC, et al. Simultaneous multislice accelerated free-breathing diffusion-weighted imaging of the liver at 3T. Abdom Imaging 2015; $40: 2323-2330$

66. Boss A, Barth B, Filli L, Kenkel D, Wurnig MC, Piccirelli M, et al. Simultaneous multi-slice echo planar diffusion weighted imaging of the liver and the pancreas: optimization of signal-to-noise ratio and acquisition time and application to intravoxel incoherent motion analysis. Eur J Radiol 
2016;85:1948-1955

67. Taron J, Martirosian P, Kuestner T, Schwenzer NF, Othman A, Weiß J, et al. Scan time reduction in diffusion-weighted imaging of the pancreas using a simultaneous multislice technique with different acceleration factors: how fast can we go? Eur Radiol 2018;28:1504-1511

68. Oshio K, Feinberg DA. GRASE (gradient- and spin-echo) imaging: a novel fast MRI technique. Magn Reson Med 1991;20:344-349

69. Feinberg DA, Oshio K. GRASE (gradient- and spin-echo) MR imaging: a new fast clinical imaging technique. Radiology
1991;181:597-602

70. Nam JG, Lee JM, Kang HJ, Lee SM, Kim E, Peeters JM, et al. GRASE revisited: breath-hold three-dimensional (3D) magnetic resonance cholangiopancreatography using a gradient and spin echo (GRASE) technique at 3T. Eur Radiol 2018;28:37213728

71. Yoshida M, Nakaura T, Inoue T, Tanoue S, Takada S, Utsunomiya D, et al. Magnetic resonance cholangiopancreatography with GRASE sequence at 3.0T: does it improve image quality and acquisition time as compared with 3D TSE? Eur Radiol 2018;28:2436-2443 